\title{
Valuing the Guaranteed Minimum Death Benefit Clause with Partial Withdrawals
}

\author{
A. C. Bélanger*, P. A. Forsyth ${ }^{\dagger}$ and G. Labahn ${ }^{\ddagger}$
}

January 30, 2009

\begin{abstract}
In this paper we give a method for computing the fair insurance fee associated with the guaranteed minimum death benefit (GMDB) clause included in many variable annuity contracts. We allow for partial withdrawals, a common feature in most GMDB contracts, and determine how this affects the GMDB fair insurance charge. Our method models the GMDB pricing problem as an impulse control problem. The resulting quasi-variational inequality is solved numerically using a fully implicit penalty method. The numerical results are obtained under both constant volatility and regime-switching models. A complete analysis of the numerical procedure is included. We show that the discrete equations are stable, monotone and consistent and hence obtain convergence to the unique, continuous viscosity solution, assuming this exists. Our results show that the addition of the partial withdrawal feature significantly increases the fair insurance charge for GMDB contracts.
\end{abstract}

Keywords: Variable annuities, guaranteed minimum death benefit (GMDB), viscosity solution, impulse control, fully implicit penalty method

Acknowledgment: This work was supported by the Natural Sciences and Engineering Research Council of Canada.

\section{Introduction}

A variable annuity or equity-linked insurance contract is a retirement and/or investment vehicle created by insurance companies. It is a contract between the customer and the insurance company where the insurer generally agrees to make periodic payments to the client starting at a given date. These contracts may also include a death benefit. Specific examples of variable annuity contracts include guaranteed minimum income benefits, guaranteed minimum withdrawal benefits $[34,20,12]$ and guaranteed minimum death benefits.

In the case of the guaranteed minimum death benefit (GMDB), if the customer passes away before the maturity of the contract, then the beneficiary receives the greater of the investment

\footnotetext{
${ }^{*}$ Cheriton School of Computer Science, University of Waterloo, Waterloo, ON, Canada, N2L 3G1 (e-mail: acbelang@cs.uwaterloo.ca)

${ }^{\dagger}$ Cheriton School of Computer Science, University of Waterloo, Waterloo, ON, Canada, N2L 3G1 (e-mail: paforsyt@cs.uwaterloo.ca)

${ }^{\ddagger}$ Cheriton School of Computer Science, University of Waterloo, Waterloo, ON, Canada, N2L 3G1 (e-mail: glabahn@cs.uwaterloo.ca)
} 


\footnotetext{
${ }^{1}$ Intuitively, this can be viewed as a discretely observed lookback option based on the maximum value of the underlying [41].
} account value or the death benefit. We consider the case of market guarantees, where some form of market returns are guaranteed through periodic ratchet dates [35]. A GMDB contract has two phases: the accumulation phase and the continuation phase. During the accumulation phase, the value of the death benefit is reset periodically to the maximum of the current account value or the prior death benefit value ${ }^{1}$. Once the accumulation phase is over, the continuation phase begins with the value of the death benefit now remaining constant. The contract usually expires when the client turns a certain age (e.g. 90) or else when the client passes away.

A common feature in GMDB contracts is the ability to have partial withdrawals from the account. Determining the fair insurance fee for a GMDB contract allowing partial withdrawals is a challenging and important problem. The stochastic nature of the contract maturity caused by the death benefit wih the market guarantees exposing insurance companies to considerable risk during prolonged periods of weak equity markets. Allowing for partial withdrawal of funds introduces a second level of uncertainty to these contracts. As discussed in [18], a conservative approach to pricing these guarantees is based on assuming optimal withdrawal at any given instant (i.e. the worst case from the hedger's point of view). Thus determining insurance fees for GMBD contracts with partial withdrawal becomes an optimal control problem.

GMDB contracts have been particularly popular in the United States and the United Kingdom since the investment gains are tax-deferred until the funds are withdrawn or annuitized at retirement. Their popularity along with the recent market turmoil has highlighted the importance of correctly pricing and hedging these complex contracts. As an example, poor hedging of variable annuities has caused large mark-to-market losses for insurance companies [10, 25].

Bauer, Kling and Russ [7] give a solution to the GMDB problem allowing optimal withdrawal at discrete instances under a constant volatility Brownian motion pricing model. In between the withdrawal times, the solution of a modified Black-Scholes PDE is determined by a Green's function integral, which is approximated numerically. The optimal withdrawal at each withdrawal time is determined by a grid search. Other methods for pricing GMDB contracts but without partial withdrawals can be found in $[33,24,15]$.

The main results of this paper are

- We determine the fair insurance charge for a GMDB contract from a combined no-arbitrage and actuarial approach (see [43]). We characterize the GMDB pricing problem as an impulse control problem and develop a pricing model based on partial differential inequalities. We use a regime switching model $[8,21,28]$ for the underlying stochastic process. Regime switching is considered to be a realistic model for long term contracts, while being more parsimonius than a stochastic volatility model with jumps.

- Our valuations for the fair insurance fee of GMDB contracts are determined as solutions to a four dimensional system of nonlinear PDEs. This nonlinear system is solved using a fully implicit penalty method, where we allow both complete lapsation and partial withdrawal. We take care to ensure that our discretization converges to the unique viscosity solution [19] between rachet dates. It is well known that the viscosity solution is the financially relevant solution of option pricing problems.

- Our results show that the the withdrawal feature is very valuable and results in significantly higher insurance fees than found previously in the literature when withdrawals are ignored. 
Due to the recent drop in equity markets, these guarantees are now substantially in the money. If these guarantess have not been hedged correctly, large mark-to-market losses will ensue.

Unlike previous work mentioned above, our approach gives a complete solution to the GMDB problem with partial withdrawal. By this we mean that we: (a) give a complete specification of the problem in terms of PDEs, including localized boundary conditions; (b) discretize the system of PDEs using a fully implicit method; and (c) prove that the discrete equations converge to the viscosity solution [19] (assuming it exists) away from ratchet dates. The last named property follows from proving that our discrete equations are monotone, stable and consistent.

While we have looked at a particular pricing problem which results in an impulse control problem, such problems occur naturally in many other financial contexts. We expect that our techniques, along with the ability to obtain provably correct solutions, will generalize to other impulse control problems in finance.

The remainder of this paper is organized as follows. In Section 2, we give the model for pricing GMDB contracts with constant volatility in terms of an impulse control problem. The pricing model is then extended in Section 3 to include the concept of regime-switching with Section 4 detailing the boundary conditions. Section 5 outlines details of the numerical solution method, while Section 6 contains a theoretical analysis of the discrete pricing model. Proofs justifying the theory are given in the following section. Numerical results obtained when computing the no-arbitrage insurance charge for the GMDB guarantee are presented in Section 7. Concluding remarks are made in Section 8. The appendix contains descriptions of GMDB contracts needed to construct our pricing model along with some technical details of the proofs.

\section{Pricing the GMDB with Partial Withdrawls Problem}

The cost to the issuer of a GMDB guarantee can be modelled as a function of four variables $V=V(S, B, D, t)$ with $t$ being time and:

- $S$ is the current value of the underlying investment account,

- $B$ is the current death benefit level,

- $D$ is the current amount deposited in the investment account.

For ease of exposition, we will first consider the no-arbitrage valuation of the GMBD under the Black-Scholes framework. We ignore the possibility of partial withdrawal for the moment. Recall that a typical GMDB contract provides market guarantees by locking in gains at ratchet dates. At each ratchet date, the death benefit $B$ is reset to the maximum of the current benefit and the investment account $S$. No-arbitrage implies that for any rachet date $t_{o}$ we have

$$
V\left(S, B^{+}, D, t_{o}^{+}\right)=V\left(S, B^{-}, D, t_{o}^{-}\right)
$$

where $B^{+}=\max \left(B^{-}, S\right)$ and $t_{o}^{-}$and $t_{o}^{+}$are times just before and after $t_{o}$. As such we only need determine the prices away from the rachet dates.

We assume that the underlying $S$ follows a classic geometric Brownian motion process (under the risk-neutral measure)[29]:

$$
\frac{d S}{S}=\left(r-\rho_{\text {total }}\right) d t+\sigma d Z
$$


Here $r$ is the risk-free rate, $\rho_{\text {total }}$ are the mortality and expense (M\&E) fees, $\sigma$ is the asset volatility and $d Z$ is the increment of a Wiener process [41].

We remark that the annual fees $\rho_{\text {total }}$ associated with variable annuity contracts, are charged to the policy owner. These fees are calculated as a predetermined percentage of the account value $S$, and include both management fees $\left(\rho_{\text {man }}\right)$ and insurance charges $\left(\rho_{\text {ins }}\right)$ so that

$$
\rho_{\text {total }}=\rho_{\text {man }}+\rho_{\text {ins }}
$$

Assuming the management fees $\left(\rho_{\text {man }}\right)$ are known, we will determine in Section 7 the value of $\rho_{\text {ins }}$ such that the issuer does not incur any loss, assuming the contract is hedged. As outlined in [35], these M\&E charges can be modeled similarly to dividends.

When the GMDB contract expires at $t=T$, the owner, if still alive, receives a payoff corresponding to the value of the invested capital at contract maturity. As such, the issuing company is not liable for any additional payment at maturity beyond the current investment account value so

$$
V(S, B, D, T)=0 .
$$

Following the derivation in [42, 43], and described in Appendix A, the cost of the GMDB guarantee in the Black-Scholes framework is then given by

$$
V_{t}+\frac{1}{2} \sigma^{2} S^{2} V_{S S}+\left(r-\rho_{\text {total }}\right) S V_{S}-r V-\mathcal{R}(t) \rho_{\text {ins }} S+\mathcal{M}(t) f=0,
$$

where $\mathcal{M}(t)$ represents the mortality function of the policy owners, $\mathcal{R}(t)$ is the survival probability of policy owners and $f=f(S, B, D, t)$ denote the death benefit exposure to the issuer. The mortality function is defined such that the fraction of original owners who pass away during the time interval $[t, t+d t]$ is $\mathcal{M}(t) d t$. Consequently, the portion of policy owners still alive at time $t$, denoted by $\mathcal{R}(t)$, is:

$$
\mathcal{R}(t)=1-\int_{0}^{t} \mathcal{M}(n) d n,
$$

where the integral term represents the owners who have died during the period $[0, t]$. Note that equation (2.5) is derived under the assumption that mortality risk is diversifiable amongst many policy owners [33]. In Appendix B we show that the death benefit $f$ is given by

$$
f(S, B, D, t)=\max (B-S, 0)+\gamma(t) D
$$

where $\gamma(t)$ is the partial or full withdrawl (lapsing) charge.

In this paper we also include a second level of uncertainty by allowing holders of GMDB contracts to withdraw some of their funds at any time. Many GMDB contracts include a feature allowing the policy owner to make partial withdrawals from the invested capital at any time prior to the maturity of the contract (during both the accumulation and continuation phase). When the owner makes a withdrawal, both the deposit $D$ and the death benefit $B$ are reduced [37]. In this work, we assume that $D$ and $B$ are reduced on a dollar-for-dollar basis following a partial withdrawal. ${ }^{2}$

In Appendix $\mathrm{C}$ we give the details showing that the pricing problem with partial withdrawals for the GMDB guarantee (away from the ratchet dates) can be given as an impulse control problem.

\footnotetext{
${ }^{2}$ We remark that our PDE approach can easily be extended to model different withdrawal policies. For example, an alternate withdrawal policy whereby the deposit is reduced by the amount withdrawn but the death benefit is reduced on a proportional basis, could be easily implemented.
} 
If we change variables to $\tau=T-t$, the time to maturity (with an abuse of notation, we now let $V=V(S, B, D, \tau), \mathcal{M}=\mathcal{M}(\tau)$, and so on), then this impulse control problem is

$$
\min \left(V_{\tau}-\mathcal{L} V+\mathcal{R}(\tau) \rho_{\text {ins }} S-\mathcal{M}(\tau) f, V-\mathcal{A} V\right)=0
$$

Here the differential operator $\mathcal{L}$ is defined as

$$
\mathcal{L} V=\frac{1}{2} \sigma^{2} S^{2} V_{S S}+\left(r-\rho_{\text {total }}\right) S V_{S}-r V
$$

while $\mathcal{A} V(S, B, D, \tau)$ given by

$\mathcal{A} V \equiv \max \left(-\mathcal{R}(\tau) \gamma(\tau) S, \max _{W \in[0, S-\omega]}(V(S-W, \max (B-W, 0), \max (D-W, 0), \tau)-\mathcal{R}(\tau) \gamma(\tau) W)-c\right)$

with $c>0$ denoting a small fixed cost added to the constraint to ensure that the impulse control problem is well-posed. The operator $\mathcal{A} V$ represents the value of the guarantee after a full or partial withdrawal.

Equation (2.8) can be interpeted in the following intuitive way. If it is optimal continue to hold the contract, then

$$
V_{\tau}-\mathcal{L} V+\mathcal{R}(\tau) \rho_{\text {ins }} S-\mathcal{M}(\tau) f=0
$$

and, since we are better off not withdrawing

$$
V-\mathcal{A} V>0
$$

Conversely, if it is optimal to withdraw assets from the account, we have

$$
V-\mathcal{A} V=0
$$

and since we are better off withdrawing rather than coninuing to hold

$$
V_{\tau}-\mathcal{L} V+\mathcal{R}(\tau) \rho_{\text {ins }} S-\mathcal{M}(\tau) f>0
$$

We can also express equation (2.8) as a penalized problem

$$
\lim _{\epsilon \rightarrow 0}\left(V_{\tau}-\mathcal{L} V+\mathcal{R}(\tau) \rho_{i n s} S-\mathcal{M}(\tau) f-\frac{1}{\epsilon} \max (\mathcal{A} V-V, 0)\right)=0 .
$$

In Section 6 we will show that a discrete version of equation (2.15) is consistent with equation 2.8 . We remark that, while our formulation requires that $c>0$, the numerical scheme presented in this paper accepts both $c=0$ and $c>0$. We expect in practice that very small values of $c$ will have little effect on the numerical solution obtained. This is confirmed by the examples included in Section 7. 


\section{Pricing the GMDB Guarantee with Regime-Switching}

Assuming that a market has constant volatility for option contracts is well-known to be inconsistent with the implied volatility observed in the market. In particular, this is totally unrealistic for options that ae based on long term horizons. At a minimum one would at least need assumptions that takes into consideration that, over a long time frame, markets will somehow alternate between high, medium and low volatility states.

In this section, we introduce the concept of regime-switching to the GMDB impulse control problem in equation (2.15). The underlying assumption with regime-switching is that the volatility switches randomly between a finite number of states or regimes. Each regime has a different volatility value and is meant to represent a different economic state. While the underlying account value follows a log-normal process within a given state, a jump in $S$ occurs when the state of the economy changes. While stochastic volatility [41] also provides a valid alternative when dealing with long-term contracts such as variable annuities, such models implies solving a higher dimensional PDE. Regime-switching appears to be less expensive from a computational point of view and is somewhat more intuitive.

Introduced in [27], the concept of regime-switching has since been used extensively when modeling both interest rates $[26,45,14]$ and pricing option contracts $[8,21,46,11,9]$. Regime switching models have also been suggested for use in long term insurance contracts [28]. These models allow for a parsimonius model which takes into account the fact that the economy typically alternates between high, medium and low volatility states. It is straightforward to incorporate long-term views about different states of the economy with a regime switching model, possibly employing both market and historical data. This contrasts with the use of a local volatility model, which is usually calibrated to short term market data, and is of questionable applicability for long term contracts.

To extend our modelling framework to regime-switching, we introduce an additional modeling variable $E$ which represents the current state of the economy and define $M$ distinct states: $E \in\left\{e_{1}, e_{2}, \ldots, e_{M}\right\}$. Associated with each state $e_{m}$ is a constant volatility value denoted as $\sigma_{m}$. Assuming we are in state $e_{m}$, the value of the GMDB guarantee is denoted as:

$$
V^{m}=V\left(S, B, D, e_{m}, t\right) .
$$

For a given regime $e_{m}$, the value of the underlying investment account $S$ follows (under the risk neutral measure):

$$
\frac{d S}{S}=\left(r-\rho_{\text {total }}-\sum_{\substack{l=1 \\ l \neq m}}^{M} \lambda^{m \rightarrow l}\left(J^{m \rightarrow l}-1\right)\right) d t+\sigma_{m} d Z+\sum_{\substack{l=1 \\ l \neq m}}^{M}\left(J^{m \rightarrow l}-1\right) d q^{m \rightarrow l},
$$

where $d q^{m \rightarrow l}$ is an independent Poisson process and $J^{m \rightarrow l} \geq 0(l \neq m)$ is an impulse function producing a jump from $S$ to $J^{m \rightarrow l} S$ when the state of the economy changes from $e_{m}$ to $e_{l}$. We define $\lambda^{m \rightarrow l}(l \neq m)$ as the risk-neutral probability of a jump from economic state $e_{m}$ to state $e_{l}$ and have $($ for $l \neq m$ ):

$$
d q^{m \rightarrow l}= \begin{cases}0 & \text { with probability } 1-\lambda^{m \rightarrow l} d t \\ 1 & \text { with probability } \lambda^{m \rightarrow l} d t\end{cases}
$$


Equation (3.8) can also be written in penalized form:

$$
\lim _{\epsilon \rightarrow 0}\left(V_{\tau}^{m}-\mathcal{L} V^{m}+\mathcal{R}(\tau) \rho_{i n s} S-\mathcal{M}(\tau) f-\frac{1}{\epsilon} \max \left(\mathcal{A} V^{m}-V^{m}, 0\right)\right)=0 .
$$


This set of coupled PDEs is solved, working backward in time, using an iterative penalty scheme [23] to determine the value of the guarantee at each timestep. See [22] for a description of the iterative method and a proof of convergence.

\section{Boundary Conditions}

For each regime $e_{m}$, the GMDB guarantee pricing problem in equation (3.10) is solved on an $S \times B \times D \times \tau$ domain. Since $B=\mathcal{D}_{0}$ at $\tau=T$ (or $t=0$ ), equation (2.1) indicates that the benefit level $B$ can only increase, unless a withdrawal occurs. Similarly, $D=\mathcal{D}_{0}$ at $\tau=T$ and the deposit $D$ decreases only when a partial withdrawal occurs. Since $D$ is reduced by the same amount as $B$ following a withdrawal, we have that $B \geq D$ and so the solution domain is

$$
[0, \infty] \times[D, \infty] \times\left[0, \mathcal{D}_{0}\right] \times[0, T]
$$

where $\mathcal{D}_{0}$ is the initial investment deposit and $T$ is the contract maturity. For numerical purposes, we localize the problem to the following domain

$$
\left[0, S_{\max }\right] \times\left[D, B_{\max }\right] \times\left[0, \mathcal{D}_{0}\right] \times[0, T] .
$$

To localize the GMDB pricing problem, additional boundary conditions are necessary. As $S \rightarrow$ 0 , the partial withdrawal policy is no longer applicable and the penalized problem in equation (3.10) reduces to (noting the definition of $f=f(S, B, D, \tau)$ in equation (2.7)):

$$
V_{\tau}^{m}+r V^{m}-\mathcal{M}(\tau)(B+\gamma(\tau) D)=0 .
$$

As $S \rightarrow S_{\max }$, we make the common assumption that $V_{S S}^{m} \rightarrow 0$ [44], which implies that $V^{m}$ is a linear function of $S$, along with the additional assumption that the linear term dominates in size (see Appendix D). In the case when the state of the economy does not change then using the above assumptions, we obtain the following approximation to equation (3.10):

$$
V_{\tau}^{m}+\rho_{\text {total }} V^{m}+\mathcal{R}(\tau) \rho_{\text {ins }} S-\frac{1}{\epsilon} \max \left(\mathcal{A} V^{m}-V^{m}, 0\right)=0 ; S=S_{\text {max }} .
$$

However the presence of jumps in $S$ when the state of the economy changes requires careful consideration when $S \rightarrow S_{\max }$. More specifically, the case when $S$ jumps outside the discrete domain following a regime change, i.e. $S J^{m \rightarrow l}>S_{\max }$, must be dealt with in an appropriate manner. We assume that any asset value that jumps outside the discrete $S$ grid is set to $S_{\max }$, which implies that the jump size $J^{m \rightarrow l}(l \neq m)$ is now a function of $S$ :

$$
J^{m \rightarrow l}(S)= \begin{cases}J^{m \rightarrow l} & \text { when } 0 \leq S \leq \frac{S_{\max }}{J^{m \rightarrow l}}, \\ \frac{S_{\max }}{S} & \text { when } \frac{S_{\max }}{J^{m \rightarrow l}}<S \leq S_{\max } .\end{cases}
$$

Again, this is an approximation, where we expect the error to be small as $S_{\max } \rightarrow \infty$. This will be verified in some numerical tests in Section 7. This new dependence of the jump size on $S$ is one of complications that need to be addressed when our discretization is analyzed for stabilty and convergence to the expected solution.

The penalized GMDB pricing equation with regime-switching can then be written as:

$$
V_{\tau}^{m}-\mathcal{L} V^{m}+\mathcal{R}(\tau) \rho_{i n s} S-\mathcal{M}(\tau) f-\frac{1}{\epsilon} \max \left(\mathcal{A} V^{m}-V^{m}, 0\right)=0,
$$


where:

$$
\begin{aligned}
\mathcal{L} V^{m}=\frac{1}{2} \sigma_{m}^{2} S^{2} V_{S S}^{m} & +\left(r-\rho_{\text {total }}-\sum_{\substack{l=1 \\
l \neq m}}^{M} \lambda^{m \rightarrow l}\left(J^{m \rightarrow l}(S)-1\right)\right) S V_{S}^{m}-r V^{m} \\
& +\sum_{\substack{l=1 \\
l \neq m}}^{M} \lambda^{m \rightarrow l}\left(V\left(J^{m \rightarrow l}(S) S, B, D, e_{l}, \tau\right)-V^{m}\right) .
\end{aligned}
$$

As $B \rightarrow D$, no additional boundary condition is required and the pricing equation in (3.10) is solved. As $B \rightarrow B_{\max }$, equation (3.10) is solved but the jump condition in equation (2.1) needs to be modified to take into consideration the discrete solution domain. For those grid nodes where $S>B_{\max }$, the discrete $S \times B$ plane does not contain the required data to calculate the jump condition outlined in equation (2.1). We assume that no ratchet events occur for those nodes where $S>B_{\max }$, which implies (in terms of $\tau=T-t$ ):

$$
V\left(S, B, D, e_{m}, \tau_{o}^{+}\right)= \begin{cases}V\left(S, B, D, e_{m}, \tau_{o}^{-}\right) & \text {if } S \leq B \\ V\left(S, S, D, e_{m}, \tau_{o}^{-}\right) & \text {if } B<S \leq B_{\max } \\ V\left(S, B, D, e_{m}, \tau_{o}^{-}\right) & \text {if } S>B_{\max }\end{cases}
$$

where $\tau_{o}$ denotes the ratchet date, while $\tau_{o}^{-}$and $\tau_{o}^{+}$denote the instants immediately before and after a ratchet event. This is clearly an approximation but the resulting error will be small, assuming $B_{\max }$ is chosen sufficiently large. Numerical tests conducted in Section 7 verify this to be the case.

In the $D$ direction, no additional boundary condition is required as $D \rightarrow \mathcal{D}_{0}$, since $\mathcal{A} V^{m}$ requires information only from problems where $D<\mathcal{D}_{0}$ (from equation $(2.10)$ ). As $D \rightarrow 0$, the partial withdrawal feature remains applicable and the usual pricing equation (3.10) is solved.

The boundary conditions for each regime can therefore be summarized as

$$
\begin{gathered}
V_{\tau}^{m}+r V^{m}-\mathcal{M}(\tau)(B+\gamma(\tau) D)-\sum_{\substack{l=1 \\
l \neq m}}^{M} \lambda^{m \rightarrow l}\left(V\left(0, B, D, e_{l}, \tau\right)-V^{m}\right)=0 \text { for } S=0 \\
V_{\tau}^{m}+\mathcal{R}(\tau) \rho_{\text {ins }} S+\rho_{\text {total }} V^{m}-\sum_{\substack{l=1 \\
l \neq m}}^{M} \lambda^{m \rightarrow l} J^{m \rightarrow l}(S)\left(V\left(S, B, D, e_{l}, \tau\right)-V^{m}\right) \\
\quad-\frac{1}{\epsilon} \max \left(\mathcal{A} V^{m}-V^{m}, 0\right)=0 \text { for } S=S_{\max },
\end{gathered}
$$

while the usual pricing equation in (4.6) is solved on the boundaries of the $B \times D$ plane.

\section{$5 \quad$ Numerical Solution of the GMDB Problem with Regime-Switching}

In this section, we present details for the numerical solution of the GMDB pricing problem. This includes the description of the discrete equations for the GMDB pricing problem and how the local optimization problem is handled when determining the value of the partial withdrawal constraint. 


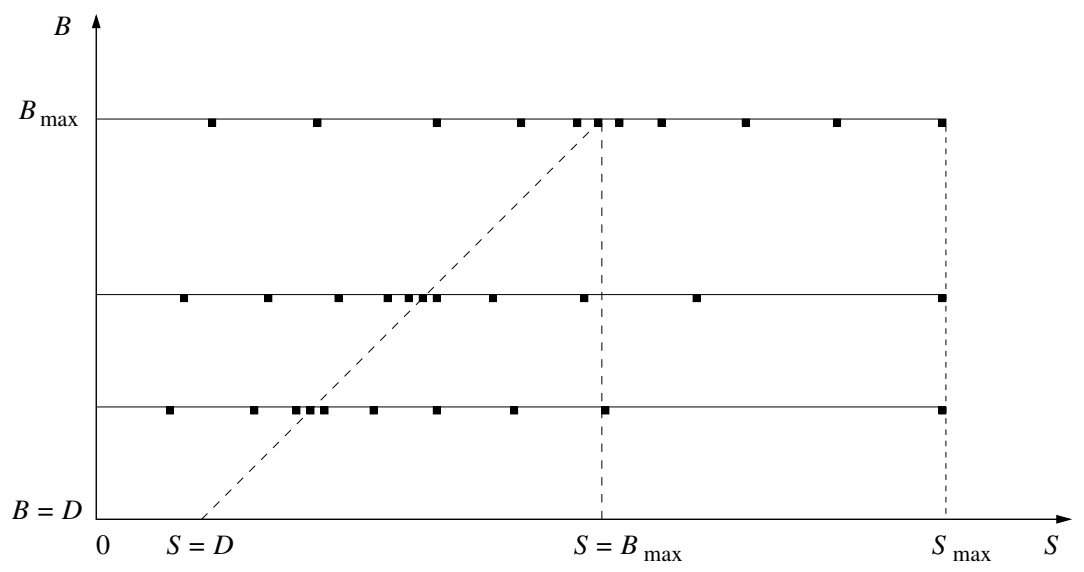

Figure 5.1: Representation of a $\left[0, S_{\max }\right] \times\left[D, B_{\max }\right]$ plane where each one-dimensional $S$ grid is built using the scaled grid technique defined in equation (5.2).

\subsection{Discrete Equations}

The discretization of equation (3.8) on the $S \times B \times D \times E$ domain follows the standard techniques of replacing derivatives by difference approximations. The discretization takes place for a sequence of four dimensional points $\left(S_{i}^{j}, B_{j}^{k}, D_{k}, e_{m}\right)$ where for each economic state $e_{m}$ we have identical grids in $\left[0, S_{\max }\right] \times\left[D, B_{\max }\right] \times\left[0, \mathcal{D}_{0}\right]$. Each such grid is build using a set of discrete values $\left\{B_{l}\right\}$ for $l=0, \ldots, l_{\max }$ in the $B$ direction and $\left\{D_{k}\right\}$, for $k=0, \ldots, k_{\max }$ in the $D$ direction. Here $B_{0}=0, B_{l_{\max }}=B_{\max }, D_{0}=0$ and $D_{k_{\max }}=\mathcal{D}_{0}$, where $\mathcal{D}_{0}$ is the initial deposit made by the policy owner. We also build the grid so that $\left\{D_{k}\right\} \subset\left\{B_{l}\right\}$, that is, each of the discrete deposit levels has a corresponding benefit level, and that the bulk of the nodes in $\left\{B_{l}\right\}$ are placed around the initial deposit amount $\mathcal{D}_{0}$..

For each state $e_{m}$ and each discrete deposit level $D_{k}$, the grid points $B_{j}^{k}$ for $j=0, \ldots, j_{\max }$ along the $B$ direction are given by

$$
B_{j}^{k}=B_{p+j} \quad \text { for } j=0, \ldots, j_{\max } .
$$

where $p$ is the value such that $B_{p}=D_{k}$. For each discrete benefit level $B_{l}$ the grid points $S_{i}^{\ell}$ for $i=0, \ldots, \ell_{\max }$ along the $S$ direction are given by

$$
S_{i}^{l}=B_{i} \frac{B_{l}}{\mathcal{D}_{0}} \quad \text { for } i=0, \ldots, l_{\max }-1 \text { and } \quad S_{i_{\max }}^{l}=\frac{\left(B_{l_{\max }}\right)^{2}}{\mathcal{D}_{0}} .
$$

The grid construction ensures that we use the minimum number of nodes to solve the GMDB pricing problem for each economic state $e_{m}$. In addition, the grid construction defined in equation (5.2) has the characteristic hat the bulk of the nodes in the $S$ direction are placed around the current benefit level $B_{l}$. This scaled grid construction enables a more precise calculation of the jump condition in equation (4.8). Note that interpolation is generally required when calculating the jump condition in (4.8) on a scaled grid. The resulting $S \times B$ grid for a fixed deposit amount $D_{k}$ is shown in Figure 5.1 and the final three-dimensional domain for a fixed economic state $e_{m}$ is given in Figure 5.2. 


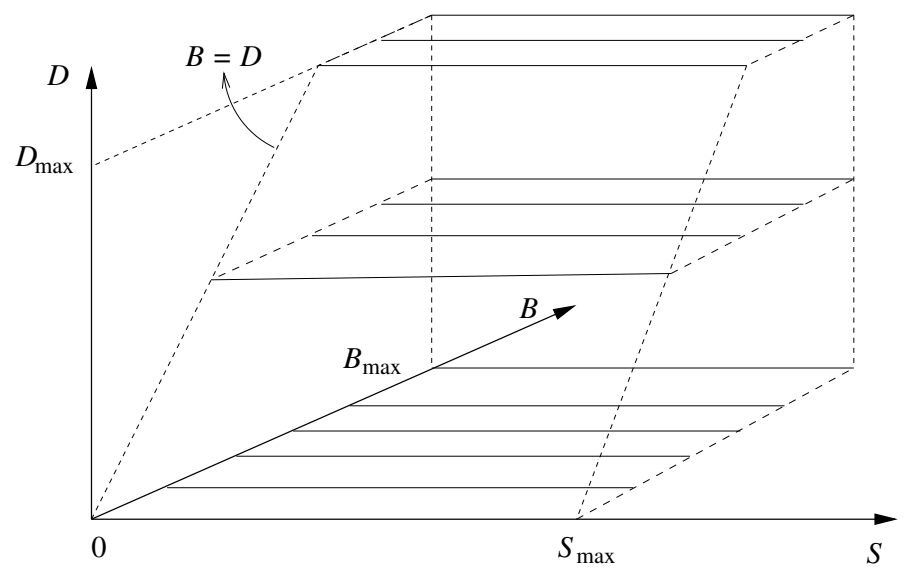

Figure 5.2: Three dimensional solution domain to price the GMDB guarantee in economic state $e_{m}$. Each $S \times B$ plane is constructed as in Figure 5.1.

Denote $V_{i, j, k, m}^{n+1}=V\left(S_{i}^{j}, B_{j}^{k}, D_{k}, e_{m}, \tau^{n+1}\right)$, and $\mathcal{A}^{h} V_{i, j, k, m}^{n+1}=\mathcal{A} V\left(S_{i}^{j}, B_{j}^{k}, D_{k}, e_{m}, \tau^{n+1}\right)$ as the discrete values and discrete version of the withdrawal constraint defined in equation (3.5), respectively. In terms of notation, discrete operators will be denoted as $\mathcal{A}^{h}$ and $\mathcal{L}^{h}$ where the superscript $h$ represents the space discretization parameter.

Assuming fully implicit timestepping is used, the discrete form of equation (3.8) is obtained by applying standard finite difference approximations:

$$
\frac{V_{i, j, k, m}^{n+1}-V_{i, j, k, m}^{n}}{\Delta \tau}=\left[\mathcal{L}^{h} V\right]_{i, j, k, m}^{n+1}-\mathcal{R}^{n+1} \rho_{i n s} S_{i}^{j}+\mathcal{M}^{n+1} f_{i, j, k}^{n+1}+\frac{\mu_{i, j, k, m}^{n+1}}{\epsilon}\left(\mathcal{A}^{h} V_{i, j, k, m}^{n+1}-V_{i, j, k, m}^{n+1}\right),
$$

where

$$
\begin{aligned}
\mathcal{M}^{n+1} & =\mathcal{M}\left(\tau^{n+1}\right), \quad \mathcal{R}^{n+1}=\mathcal{R}\left(\tau^{n+1}\right), \quad \gamma^{n+1}=\gamma\left(\tau^{n+1}\right), \\
f_{i, j, k}^{n+1} & =f\left(S_{i}^{j}, B_{j}^{k}, D_{k}, \tau^{n+1}\right)=\max \left(B_{j}^{k}-S_{i}^{j}, 0\right)+\gamma^{n+1} D_{k},
\end{aligned}
$$

and

$$
\mu_{i, j, k, m}^{n+1}= \begin{cases}1 & \text { if } \mathcal{A}^{h} V_{i, j, k, m}^{n+1}>V_{i, j, k, m}^{n+1} \\ 0 & \text { otherwise }\end{cases}
$$

The discrete differential operator $\mathcal{L}^{h}$ can be written as:

$$
\begin{gathered}
{\left[\mathcal{L}^{h} V\right]_{i, j, k, m}^{n+1}=\alpha_{i, j, m} V_{i-1, j, k, m}^{n+1}+\beta_{i, j, m} V_{i+1, j, k, m}^{n+1}-\left(\alpha_{i, j, m}+\beta_{i, j, m}+r\right) V_{i, j, k, m}^{n+1}} \\
+\sum_{\substack{l=1 \\
l \neq m}}^{M} \lambda^{m \rightarrow l}\left(\mathcal{H}\left(J^{m \rightarrow l}\right)_{i} V_{j, k, l}^{n+1}-V_{i, j, k, m}^{n+1}\right)
\end{gathered}
$$

where $\alpha_{i, j, m}, \beta_{i, j, m}$ are defined in Appendix E and satisfy:

$$
\alpha_{i, j, m} \geq 0 ; \beta_{i, j, m} \geq 0 \quad \forall i, j, m
$$


and $\mathcal{H}\left(J^{m \rightarrow l}\right)_{i} V_{j, k, l}^{n+1}$ represents the interpolated guarantee value in regime $e_{l}$ when the asset price jumps to $J^{m \rightarrow l}(S) S$. Assuming linear interpolation is chosen, we have:

$$
\mathcal{H}\left(J^{m \rightarrow l}\right)_{i} V_{j, k, l}^{n+1}=\left(1-w_{i, j, m}\right) V_{a, j, k, l}^{n+1}+w_{i, j, m} V_{a+1, j, k, l}^{n+1},
$$

where $S_{a}^{j} \leq J^{m \rightarrow l}\left(S_{i}^{j}\right) S_{i}^{j} \leq S_{a+1}^{j}$ and the interpolation weight $0 \leq w_{i, j, m} \leq 1$ can be written as:

$$
w_{i, j, m}=\frac{J^{m \rightarrow l}\left(S_{i}^{j}\right) S_{i}^{j}-S_{a}^{j}}{S_{a+1}^{j}-S_{a}^{j}}
$$

Since the node $\left(S_{i}^{j}-W, \max \left(B_{j}^{k}-W, 0\right), \max \left(D_{k},-W, 0\right)\right)$ does not always coincide with an existing grid node, interpolation must be used when calculating the discrete withdrawal constraint $\mathcal{A}^{h} V_{i, j, k, m}^{n+1}$. We define the vector $\mathcal{I}(W)_{i, j, k}$ as the interpolation operator used when calculating the value of the GMDB guarantee following a withdrawal $W$. Thus, we have:

$$
\mathcal{A}^{h} V_{i, j, k, m}^{n+1}=\max \left(-R^{n+1} \gamma^{n+1} S_{i}^{j}, \max _{W \in\left[0, S_{i}^{j}-\omega\right]}\left[\mathcal{I}(W)_{i, j, k} V_{m}^{n+1}-R^{n+1} \gamma^{n+1} W\right]-c\right),
$$

where $V_{m}^{n+1}$ is a vector containing the GMDB values for regime $e_{m}$ :

$$
V_{m}^{n+1}=\left[\begin{array}{c}
V_{0,0,0, m}^{n+1} \\
V_{1,0,0, m}^{n+1} \\
\vdots \\
V_{i_{\max }^{n+1}-1, j_{\max }, k_{\max }, m}^{n+1} \\
V_{i_{\max }, j_{\max }, k_{\max }, m}^{n+1}
\end{array}\right],
$$

and $\mathcal{I}(W)_{i, j, k}$ can be written as follows assuming linear interpolation:

$$
\mathcal{I}(W)_{i, j, k} V_{m}^{n+1}=\sum_{u, v, w} \eta_{u, v, w, m} V_{u, v, w, m}^{n+1},
$$

where $0 \leq \eta_{u, v, w, m} \leq 1$ are the interpolation weights and:

$$
\sum_{u, v, w} \eta_{u, v, w, m}=1
$$

Letting $W_{i, j, k, m}^{n+1}$ denote the optimal withdrawal amount at node $\left(S_{i}^{j}, B_{j}^{k}, D_{k}, e_{m}\right)$ and time $\tau^{n+1}$, and defining the indicator variable $a_{i, j, k, m}^{n+1}$ as:

$$
a_{i, j, k, m}^{n+1}= \begin{cases}1 & \text { if it is optimal to lapse, } \\ 0 & \text { if it is optimal to withdraw } W_{i, j, k, m}^{n+1},\end{cases}
$$

we can rewrite equation (5.11) as:

$$
\mathcal{A}^{h} V_{i, j, k, m}^{n+1}=-a_{i, j, k, m}^{n+1} R^{n+1} \gamma^{n+1} S_{i}^{j}+\left(1-a_{i, j, k, m}^{n+1}\right)\left(\mathcal{I}\left(W_{i, j, k, m}^{n+1}\right)_{i, j, k} V_{m}^{n+1}-R^{n+1} \gamma^{n+1} W_{i, j, k, m}^{n+1}-c\right) .
$$

The numerical scheme in equation (5.3) is a positive coefficient discretization [22] when the following definition is satisfied. 
Definition 5.1 (Positive Coefficient Scheme). The numerical scheme defined in equation (5.3) is a positive coefficient discretization when:

$$
\begin{aligned}
\alpha_{i, j, m}, \beta_{i, j, m} & \geq 0, \quad \forall i, j, m, \\
r & \geq 0, \\
\lambda^{m \rightarrow l} & \geq 0, \quad \text { when } m \neq l,
\end{aligned}
$$

and the interpolation operators $\mathcal{H}\left(J^{m \rightarrow l}\right)_{i}$ and $\mathcal{I}(W)_{i, j, k}$ represent linear interpolation.

Since $\alpha_{i, j, m}, \beta_{i, j, m} \geq 0$ by construction (see Appendix E), $\lambda^{m \rightarrow l} \geq 0$, when $m \neq l$ and $r \geq 0$ for all problems considered, the numerical scheme in (5.3) is a positive coefficient scheme.

Remark 5.2. The nonlinear discrete equations (5.3) can be solved using a policy type iteration, a method which is guaranteed to converge for any initial iterate (see [22]).

\subsection{Optimal Withdrawal}

At each discrete grid node $\left(S_{i}^{j}, B_{j}^{k}, D_{k}, e_{m}\right)$ we need to determine the optimal withdrawal $W$ when calculating the constraint in equation (3.5). This local optimization problem is solved by considering all possible discrete withdrawals. This is done by first checking that a withdrawal is possible by verifying $S_{i}^{j}>\omega$, where $\omega$ is the minimal deposit amount, and then carrying out a linear search over all possible discrete withdrawals $\bar{W}$. Here

$$
\bar{W}=\min \left(S_{l}^{j}, S_{i}^{j}-\omega\right),
$$

assuming $S_{l}^{j}<S_{i}^{j}$. For each $\bar{W}$ considered, we calculate the effect of the partial withdrawal to the issuer, denoted by $A(\bar{W})$ :

$$
A(\bar{W})=\mathcal{I}(\bar{W})_{i, j, k} V_{m}^{n+1}-\mathcal{R}^{n+1} \gamma^{n+1} \bar{W},
$$

where $\mathcal{I}(\bar{W})_{i, j, k}$ is defined in (5.13).

The optimal withdrawal is determined by taking the maximum of $A(\bar{W})$ over all discrete withdrawals $\bar{W}$ and the final withdrawal constraint for node $\left(S_{i}^{j}, B_{j}^{k}, D_{k}, e_{m}\right)$ is computed as

$$
\mathcal{A}^{h} V_{i, j, k, m}^{n+1}=\max \left(-\mathcal{R}^{n+1} \gamma^{n+1} S_{i}^{j}, \max _{\bar{W}}[A(\bar{W})]-c\right) .
$$

This search procedure is summarized in Algorithm 5.1.

\section{Convergence to the Viscosity Solution}

In [38], the authors demonstrate how some reasonable discretization schemes either never converge or converge to a wrong solution. Thus, it is important to ensure that our discretization method converges to the unique viscosity solution [19], which corresponds to the financially relevant solution. Assuming that a unique, continuous viscosity solution to equation (5.3) exists, the numerical scheme in (5.3) converges to the viscosity solution away from the ratchet dates if it satisfies certain stability, consistency and monotonicity requirements $[4,6]$. 


$$
\begin{aligned}
& \bar{W}=0 ; A=0 ; A_{\max }=0 \\
& \text { if } S_{i}^{j}>\omega \text { then }
\end{aligned}
$$

Determine maximum withdrawal: $\bar{W}=S_{i}^{j}-\omega$

Calculate: $A_{\max }=\mathcal{I}(\bar{W})_{i, j, k} V_{m}^{n+1}-\mathcal{R}^{n+1} \gamma^{n+1} \bar{W}$

Determine index $i_{\max }$ s.t.: $S_{i-1}^{j}<S_{i_{\max }}^{j}<S_{i}^{j}-\omega$

for $l=0, \ldots, i_{\max }$ do

Determine withdrawal: $\bar{W}=S_{l}^{j}$

Calculate: $A=\mathcal{I}(\bar{W})_{i, j, k} V_{m}^{n+1}-\mathcal{R}^{n+1} \gamma^{n+1} \bar{W}$

$A_{\max }=\max \left(A, A_{\max }\right)$

\section{end for}

\section{end if}

$$
\mathcal{A}^{h} V_{i, j, k}^{n+1}=\max \left(A_{\max }-c,-\mathcal{R}^{n+1} \gamma^{n+1} S_{i}^{j}\right)
$$

\section{Algorithm 5.1: Calculation of Withdrawal Constraint for GMDB Contracts}

Assuming a given state $e_{m}$, the solution domain for the GMDB pricing problem in equation (3.8) is $\left[0, S_{\max }\right] \times\left[D, B_{\max }\right] \times\left[0, \mathcal{D}_{0}\right]$. When working backward in time, we denote the ratchet dates as $\tau_{o}^{u}$ for $u=0, \ldots, u_{\max }$, and use $\tau_{o}^{u-}$ and $\tau_{o}^{u+}$ to denote the times right before and after a ratchet event. Thus, we define the solution domains $\Pi_{u}$ and $\Pi$ by:

$$
\begin{aligned}
\Pi_{u} & =\left[0, S_{\max }\right] \times\left[D, B_{\max }\right] \times\left[0, \mathcal{D}_{0}\right] \times\left[\tau_{o}^{u+}, \tau_{o}^{(u+1)-}\right] \text { for } u=0, \ldots, u_{\max }-1, \text { and } \\
\Pi & =\bigcup_{u} \Pi_{u}=\left[0, S_{\max }\right] \times\left[D, B_{\max }\right] \times\left[0, \mathcal{D}_{0}\right] \times \bigcup_{u}\left[\tau_{o}^{u+}, \tau_{o}^{(u+1)-}\right] .
\end{aligned}
$$

This enables us to define the pricing problem for the GMDB guarantee in detail.

Definition 6.1 (GMDB Pricing Problem with Discrete Ratchets). The pricing problem for the $G M D B$ guarantee with discrete ratchet events is defined in $\Pi$ as follows: within each domain $\Pi_{u}$, for $u=0, \ldots, u_{\max }-1$, we determine the solution to the pricing problem presented in equation (3.8) with initial conditions expressed in equation (3.7) when $u=0$ or in equation (4.8) when $u>0$, boundary conditions described in equations (4.9)-(4.10) and localization conditions in equations (4.5) and (4.8).

Remark 6.2. Note that we have not defined the pricing problem for the GMDB guarantee over the entire contract lifetime $\tau \in[0, T]$ since the solution can be discontinuous across ratchet dates $\tau_{o}^{u}$, for $u=0, \ldots, u_{\max }-1$, due to the no-arbitrage condition in equation (4.8).

Assumption 6.3. We assume that a unique, continuous viscosity solution exists [4, 32, 36] for the localized pricing problem in Definition 6.1 which satisfies equations (4.9)-(4.10) and localization conditions in equations (4.5) and (4.8). More specifically, we assume that the unique viscosity solution is continuous within each domain $\Pi_{u}$, for $u=0, \ldots, u_{\max }-1$.

Remark 6.4. A unique, continuous viscosity solution exists if the PDE satisfies a strong comparison property. In a financial context, the strong comparison property states that if $U(S, \tau)$ and $V(S, \tau)$ are two contingent claims with $U(S, 0) \geq V(S, 0)$, then $U(S, \tau) \geq V(S, \tau)$ for any time 
$\tau$ [17]. Strong comparison has been shown to hold for similar (but not identical) scaler impulse control problems in [40, 1, 30]. In the regime switching case, existence of a continuous, viscosity solution is shown using properties of the value function [36]. Note that the definition of viscosity solution has to be generalized for systems of weakly coupled PDEs, such as regime switching models [32, 36].

If Assumption 6.3 holds, then showing that the discrete equations are monotone, stable and consistent will enable us to conclude that the solution of the numerical scheme in equation (5.3) converges to the unique viscosity solution of the pricing problem outlined in Definition 6.1.

\subsection{Stability}

In order to show that the discrete equations in (5.3) satisfy $l_{\infty}$-stability one needs to show that the discrete contract value $V_{i, j, k, m}^{n+1}$ is bounded. We define:

$\Delta S_{\max }^{j}=\max _{i}\left(S_{i+1}^{j}-S_{i}^{j}\right), \Delta B_{\max }^{k}=\max _{j}\left(B_{j+1}^{k}-B_{j}^{k}\right), \Delta D_{\max }=\max _{k}\left(D_{k+1}-D_{k}\right)$ and $\Delta \tau=\frac{T}{N}$.

Definition 6.5 (Stability). For fixed $S_{\max }, B_{\max }$ and $T$, the numerical scheme presented in equation (5.3) is $l_{\infty}$-stable if:

$$
\left\|V^{n}\right\|_{\infty} \leq C
$$

for $0 \leq n \leq N$, as $\Delta \tau \rightarrow 0, \max _{j} \Delta S_{\max }^{j} \rightarrow 0, \max _{k} \Delta B_{\max }^{k} \rightarrow 0, \Delta D_{\max } \rightarrow 0$ and $\epsilon \rightarrow 0$. The constant $C$ is independent of $\Delta \tau, \Delta S_{\max }^{j}, \Delta B_{\max }^{k}, \Delta D_{\max }$ and $\epsilon$.

For notational convenience, we make the following assumption.

Assumption 6.6. We assume that $\Delta B_{\max }^{k}, \Delta S_{\max }^{j}, \Delta \tau$ and $\epsilon$ are parametrized as

$$
\Delta B_{\max }^{k}=c_{0} h, \Delta S_{\max }^{j}=c_{1} h, \Delta \tau=c_{2} h \text { and } \epsilon=c_{3} h,
$$

with $c_{0}, c_{1}, c_{2}$ and $c_{3}$ constants.

Theorem 6.7. Assume the numerical scheme satisfies Definition 5.1, that the boundary conditions are described by the discrete version of equations (4.9)-(4.10), that the initial conditions are given by the discrete version of equation (3.7) and that fully implicit timestepping is used. Then:

$$
-S_{i}^{j} \leq V_{i, j, k, m}^{n+1} \leq \mathcal{C}_{0}^{n+1} B_{\max }+\mathcal{C}_{1}^{n+1} D_{\max } \quad \forall i, j, k, m, n,
$$

where the constants $0 \leq \mathcal{C}_{0}^{n+1} \leq 1$ and $0 \leq \mathcal{C}_{1}^{n+1}$ are defined as:

$$
\mathcal{C}_{0}^{n+1}=\Delta \tau \sum_{i=0}^{n+1} \mathcal{M}^{i} \quad \text { and } \quad \mathcal{C}_{1}^{n+1}=\Delta \tau \sum_{i=0}^{n+1} \mathcal{M}^{i} \gamma^{i}
$$

Proof. A proof is given in Appendix F.1.

Theorem 6.7 implies that the numerical scheme for $V_{i, j, k, m}^{n+1}$, as defined in equation (5.3), is stable according to Definition 6.5. 


\subsection{Monotonicity}

In this section, we show that the discrete equations presented in (5.3) are monotone. To facilitate exposition, we denote the discrete equations on interior nodes (when $S_{i}^{j}<S_{\max }$ ) as:

$$
\begin{aligned}
\mathcal{G}\left(h, x, V_{i, j, k, m}^{n+1}, V_{i, j, k, m}^{n},\left\{V_{a, p, u, l}^{n+1}\right\}\right)=\frac{V_{i, j, k, m}^{n+1}-V_{i, j, k, m}^{n}}{\Delta \tau}- & {\left[\mathcal{L}^{h} V\right]_{i, j, k, m}^{n+1}+\mathcal{R}^{n+1} \rho_{i n s} S_{i}^{j}-\mathcal{M}^{n+1} f_{i, j, k}^{n+1} } \\
& -\frac{1}{\epsilon} \max \left(\mathcal{A}^{h} V_{i, j, k, m}^{n+1}-V_{i, j, k, m}^{n+1}, 0\right),
\end{aligned}
$$

where $x=\left(S_{i}^{j}, B_{j}^{k}, D_{k}, e_{m}, \tau^{n+1}\right), h$ is the discretization parameter, and $\left\{V_{a, p, u, l}^{n+1}\right\}$ represents all discrete nodes, other than $V_{i, j, k, m}^{n+1}$ and $V_{i, j, k, m}^{n}$, included in the discrete equations. Similarly, at the boundary when $S_{i}^{j}=S_{\max }$, the discretization is given as:

$$
\begin{aligned}
\mathcal{G}\left(h, x, V_{i_{\max }, j, k, m}^{n+1}, V_{i_{\max }, j, k, m}^{n},\left\{V_{a, p, u, l}^{n+1}\right\}\right)= & \frac{V_{i_{\max }, j, k, m}^{n+1}-V_{i_{\max }, j, k, m}^{n}}{\Delta \tau}+\rho_{\text {total }} V_{i_{\max }, j, k, m}^{n+1}+\mathcal{R}^{n+1} \rho_{i n s} S_{i_{\max }}^{j} \\
& -\sum_{\substack{l=1 \\
l \neq m}}^{M} \lambda^{m \rightarrow l} J_{i_{\max }^{m \rightarrow l}}^{m \rightarrow l}\left(V_{i_{\max }, j, k, l}^{n+1}-V_{i_{\max , j, k, m}}^{n+1}\right) \\
& -\frac{1}{\epsilon} \max \left(\mathcal{A}^{h} V_{i_{\max }, j, k, m}^{n+1}-V_{i_{\max }, j, k, m}^{n+1}, 0\right) .
\end{aligned}
$$

Definition 6.8 (Monotonicity). The numerical scheme $\mathcal{G}\left(h, x, V_{i, j, k, m}^{n+1}, V_{i, j, k, m}^{n},\left\{V_{a, p, u, l}^{n+1}\right\}\right)$ presented in equations (6.6) and (6.7) is monotone if for all $Y_{i, j, k, m}^{n} \geq V_{i, j, k, m}^{n}$ :

$$
\mathcal{G}\left(h, x, V_{i, j, k, m}^{n+1}, Y_{i, j, k, m}^{n},\left\{Y_{a, p, u, l}^{n+1}\right\}\right)-\mathcal{G}\left(h, x, V_{i, j, k, m}^{n+1}, V_{i, j, k, m}^{n},\left\{V_{a, p, u, l}^{n+1}\right\}\right) \leq 0 .
$$

Note that this definition of monotonicity is equivalent to the one presented in [4].

Theorem 6.9 (Monotone Discretization). Assuming that the discretization satisfies Condition (5.1), the numerical scheme $\mathcal{G}\left(h, x, V_{i, j, k, m}^{n+1}, V_{i, j, k, m}^{n},\left\{V_{a, p, u, l}^{n+1}\right\}\right)$ defined in equations (6.6) and (6.7), is monotone.

Proof. Notice that the numerical scheme presented in equations (6.6) and (6.7) is a positive coefficient discretization since it satisfies Condition 5.1. In [22], the authors demonstrate that a positive coefficient discretization of a control problem, such as the one considered here, is monotone. Using the same technique as in [22], it is straightforward to show that the numerical scheme presented in equations (6.6) and (6.7) is monotone and satisfies Definition 6.8.

\subsection{Consistency}

The final step in showing that our discretization converges to the viscosity solution is to show that the numerical scheme in equation (5.3) is consistent. For the GMDB pricing problem, the impulse control problem can be written in compact form as:

$$
F(V(x))=0 \text { for all } x=\left(S, B, D, e_{m}, \tau\right),
$$


where

$$
F(V(x))= \begin{cases}F_{\text {in }}(V(x)) & \text { if } S<S_{\max } \\ F_{\text {bound }}(V(x)) & \text { if } S=S_{\max }\end{cases}
$$

The continuous problem evaluated at discrete interior nodes when $S_{i}^{j}<S_{\max }$ is then:

$$
F_{i n}(V)_{i, j, k, m}^{n+1}=\left[\min \left(V_{\tau}-\mathcal{L} V+\mathcal{R}(\tau) \rho_{i n s} S-\mathcal{M}(\tau) f, V-\mathcal{A} V\right)\right]_{i, j, k, m}^{n+1}=0
$$

while at boundary nodes when $S_{i}^{j}=S_{\max }$ we have:

$$
\begin{array}{r}
F_{\text {bound }}(V)_{i_{\max }, j, k, m}^{n+1}=\left[\operatorname { m i n } \left(V_{\tau}+\rho_{\text {total }} V-\sum_{\substack{l=1 \\
l \neq m}}^{M} \lambda^{m \rightarrow l} J^{m \rightarrow l}(S)\left(V\left(S, B, D, e_{l}, \tau\right)-V\right)+\mathcal{R}(\tau) \rho_{\text {ins }} S,\right.\right. \\
V-\mathcal{A} V)]_{i_{\max }, j, k, m}^{n+1}=0, \quad(6.12)
\end{array}
$$

where the continuous operator $\mathcal{L}$ is defined in equation $(3.9)$ and $f=f(S, B, D, \tau)$ is defined in equation (2.7).

Since $\epsilon>0$, the discrete scheme in equation (6.6) can be rewritten as:

$$
\begin{gathered}
\hat{\mathcal{G}}\left(h, x, V_{i, j, k, m}^{n+1}, V_{i, j, k, m}^{n},\left\{V_{a, p, u, l}^{n+1}\right\}\right)= \\
\min \left(\epsilon\left(\frac{V_{i, j, k, m}^{n+1}-V_{i, j, k, m}^{n}}{\Delta \tau}-\left[\mathcal{L}^{h} V\right]_{i, j, k, m}^{n+1}+\mathcal{R}^{n+1} \rho_{i n s} S_{i}^{j}-\mathcal{M}^{n+1} f_{i, j, k}^{n+1}\right)+V_{i, j, k, m}^{n+1}-\mathcal{A}^{h} V_{i, j, k, m}^{n+1},\right. \\
\left.\frac{V_{i, j, k, m}^{n+1}-V_{i, j, k, m}^{n}}{\Delta \tau}-\left[\mathcal{L}^{h} V\right]_{i, j, k, m}^{n+1}+\mathcal{R}^{n+1} \rho_{i n s} S_{i}^{j}-\mathcal{M}^{n+1} f_{i, j, k}^{n+1}\right)=0,
\end{gathered}
$$

at interior nodes when $S_{i}^{j}<S_{\max }$, while equation (6.7) can be rewritten as:

$$
\begin{aligned}
& \hat{\mathcal{G}}\left(h, x, V_{i_{\max }, j, k, m}^{n+1}, V_{i_{\max }, j, k, m}^{n},\left\{V_{a, p, u, l}^{n+1}\right\}\right)=\min \left(\epsilon \left(\frac{V_{i_{\max }, j, k, m}^{n+1}-V_{i_{\max }, j, k, m}^{n}}{\Delta \tau}+\rho_{\text {total }} V_{i_{\max }, j, k, m}^{n+1}\right.\right. \\
& \left.-\sum_{\substack{l=1 \\
l \neq m}}^{M} \lambda^{m \rightarrow l} J_{i_{\max }}^{m \rightarrow l}\left(V_{i_{\max }, j, k, l}^{n+1}-V_{i_{\max }, j, k, m}^{n+1}\right)+\mathcal{R}^{n+1} \rho_{i n s} S_{i_{\max }}^{j}\right)
\end{aligned}
$$

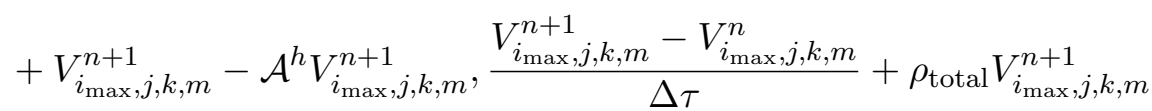

$$
\begin{aligned}
& \left.-\sum_{\substack{l=1 \\
l \neq m}}^{M}\left(\lambda^{m \rightarrow l} J^{m \rightarrow l}\right)_{i_{\max }}\left(V_{i_{\max }, j, k, l}^{n+1}-V_{i_{\max }, j, k, m}^{n+1}\right)+\mathcal{R}^{n+1} \rho_{i n s} S_{i_{\max }}^{j}\right)=0,
\end{aligned}
$$

on the boundary when $S_{i}^{j}=S_{\max }$.

To formally define the notion of consistency, we require the concept of upper and lower semicontinuous envelope of a function. 
Definition 6.10. Assume we have a function $f: C \rightarrow \mathbb{R}$ where $C$ is a topological space. Then the upper semi-continuous and lower semi-continuous envelopes of $f$ are defined as:

$$
f^{*}(y)=\lim _{\substack{x \rightarrow y \\ y \in C}} f(x) \quad \text { and } \quad f_{*}(y)=\lim _{\substack{x \rightarrow y \\ y \in C}} f(x) .
$$

Definition 6.11 (Consistency). For any smooth test function $\phi$ with bounded derivatives of all orders with respect to $S$ and $\tau$, the numerical scheme $\hat{\mathcal{G}}\left(h, x, \phi_{i, j, k, m}^{n+1}, \phi_{i, j, k, m}^{n},\left\{\phi_{a, p, u, l}^{n+1}\right\}\right)$ is consistent if, for all points in the domain $\hat{x}=\left(\hat{S}, \hat{B}, \hat{D}, e_{m}, \hat{\tau}\right)$ with $x=\left(S_{i}^{j}, B_{j}^{k}, D_{k}, e_{m}, \tau^{n+1}\right)$, we have:

$$
\begin{aligned}
& \lim \sup _{\substack{h, \xi \rightarrow 0 \\
x \rightarrow \hat{x}}} \hat{\mathcal{G}}\left(h, x, \phi_{i, j, k, m}^{n+1}+\xi, \phi_{i, j, k, m}^{n}+\xi,\left\{\phi_{a, p, u, l}^{n+1}+\xi\right\}\right) \leq F^{*}(\phi(\hat{x})), \\
& \lim \inf _{\substack{h, \xi \rightarrow 0 \\
x \rightarrow \hat{x}}} \hat{\mathcal{G}}\left(h, x, \phi_{i, j, k, m}^{n+1}+\xi, \phi_{i, j, k, m}^{n}+\xi,\left\{\phi_{a, p, u, l}^{n+1}+\xi\right\}\right) \geq F_{*}(\phi(\hat{x})),
\end{aligned}
$$

where $\phi_{i, j, k, m}^{n}=\phi\left(S_{i}^{j}, B_{j}^{k}, D_{k}, e_{m}, \tau^{n}\right)$ and $\xi \geq 0$.

Remark 6.12 (Continuous Scheme). When the numerical scheme is continuous over the entire domain (both interior nodes and boundary), the conditions in equations (6.16) and (6.17) reduce to:

$$
\lim _{h \rightarrow 0}\left|F(\phi)_{i, j, k, m}^{n+1}-\hat{\mathcal{G}}\left(h, x, \phi_{i, j, k, m}^{n+1}, \phi_{i, j, k, m}^{n},\left\{\phi_{a, p, u, l}^{n+1}\right\}\right)\right|=0 .
$$

Equation (6.18) is the typical formulation used when verifying consistency of a numerical scheme and applies, for example, to cases where the equation on the boundary is obtained by taking the limit of the equation on the interior nodes. Unfortunately, this is not the case for our GMDB pricing model which is why the consistency requirements are outlined as in equations (6.16) and (6.17).

Theorem 6.13 (Consistent Discretization). The numerical scheme presented in equation (5.3) is consistent according to Definition 6.11.

Proof. See Appendix F.2.

\section{Results from Numerical Experiments}

In the previous section we have shown that our discretization converges to the financially relevant solution for the GMDB problem allowing partial withdrawls. In this section we give some numerical results. In particular we focus on determining the fair insurance charge associated with a GMDB guarantee from the issuer's perspective. More specifically, we are looking for $\rho_{\text {ins }}$ such that:

$$
V\left(\rho_{\text {ins }} ; S=\mathcal{D}_{0}, B=\mathcal{D}_{0}, D=\mathcal{D}_{0}, E=e_{m}, \tau=T\right)=0,
$$

where $\mathcal{D}_{0}$ is the initial deposit made by the contract owner and $T$ is the contract maturity in years. Newton iteration is used to determine the fair insurance charge $\rho_{\text {ins }}$ that satisfies equation (7.1) assuming an economic state $e_{m}$. The Newton iteration tolerance, denoted by tol, ensures that:

$$
\left|\rho_{i n s}^{k+1}-\rho_{i n s}^{k}\right|_{\infty} \leq \text { tol },
$$


where $t o l=1 \times 10^{-6}$ and $k$ is the iteration index. Unless otherwise stated, this tolerance level is used for all numerical results included in this section.

Intuitively (and as seen in the numerical examples)

- If $\rho_{\text {ins }}=0$, then the value of the guarantee is strictly positive for $B>0$ if the mortality $\mathcal{M}>0$ in $[0, T]$ (this is a free guarantee).

- If $\rho_{\text {ins }}$ is sufficiently large, then the value of the guarantee is negative (since it will be optimal to withdraw and pay the surrender charge).

- The guarantee value is decreasing in $\rho_{i n s}$ (no-arbitrage).

If the above properties hold, then the Newton iteration will always converge to a unique solution. In our numerical experiments, the Newton iteration always converges (using $r h o_{i n s}=0$ as an initial estimate). However, we have no proof of these properties and this would be an interesting avenue for further research.

\subsection{Comparison with Previous Results}

We were not able to find previous work with handles the case of continuous partial and full withdrawal. In [33], an analytical solution was developed for the case with no withdrawals, continuous ratchets, no management fees, and constant volatility. This is, of course, a special case of our model. In Appendix G, we find that our results are in good agreement with the results in [33] for this special case.

\subsection{Results for Constant Volatility}

In this section we consider the simplest case where we have only one economic state $e_{0}$ and constant volatility. The volatility associated with $e_{0}$, as well as other contract parameters, are presented in Table 7.1. We are looking to determine the insurance fee $\rho_{\text {ins }}$ which satisfies:

$$
V\left(\rho_{\text {ins }} ; S=\$ 100, B=\$ 100, D=\$ 100, E=e_{0}, \tau=T\right)=0 .
$$

Additional assumptions are necessary regarding the owner of the GMDB contract. We assume that the owner of the variable annuity is a male of 50 years of age at the time of purchase. As such, the accumulation period of the contract, during which there are periodical ratchet events, will last 30 years. The contract is assumed to come to maturity when the owner turns 90 years old which implies that $T=40$ years, as reflected in Table 7.1. The mortality data used to price the GMDB guarantee is taken from the Complete life table, Canada, 1995-1997 for males and females found in $[16]$.

Table 7.1 also specifies some grid construction details. While an unequally spaced grid containing 36 nodes is built along $S$, the grid built in the $D$ direction contains 21 nodes spanning $\left[0, \mathcal{D}_{0}\right]$. Though not presented here, numerical tests were carried out to ensure that the choice of $B_{\max }$, and consequently $S_{\max }$, provides a minimum of 6 digits of accuracy. Recall that $S_{\max }=B_{\max }^{2} / \mathcal{D}_{0}$, where $\mathcal{D}_{0}$ is the initial deposit (see Section 5.1 for more details). Similarly, numerical tests show that choosing a sufficiently small fixed cost, such as $c=1 \times 10^{-10}$, results in values identical to those obtained when $c=0$ up to at least 6 digits. Consequently, for all numerical experiments in this section, we set $c=1 \times 10^{-10}$. From Theorem 6.13 we have that the discretization (5.3) is consistent 


\begin{tabular}{ll}
\hline \multicolumn{2}{c}{ State Information $-e_{0}$} \\
\hline$\sigma_{0}$ - Volatility & 0.20 \\
\hline \multicolumn{2}{c}{ Contract Information } \\
\hline$r$ - Interest rate & 0.06 \\
$\rho_{\text {man }}$ - Management fees & 0.015 \\
Ratchet interval & 1 year \\
Last Ratchet Date & 30 years \\
T - Contract maturity & 40 years \\
\hline \multicolumn{2}{c}{ Grid Construction } \\
\hline $\mathcal{D}_{0}$ - Initial deposit & $\$ 100$ \\
$S_{\max }$ - Grid parameter & $\$ 3.6 \times 10^{7}$ \\
$B_{\max }$ - Grid parameter & $\$ 60000$ \\
\hline
\end{tabular}

Table 7.1: Parameter values used when pricing the GMDB guarantee in the classic Black-Scholes context.

if the penalty parameter $\epsilon$ (see equations (6.6) and (6.7)) is $\epsilon=\Delta \tau C_{1}$ for any $C_{1}>0$. In practice, in order to obtain reasonable results for finite $\Delta \tau$, we use $C_{1}=10^{-6}$. Using $C_{1} \in\left[10^{-4}, 10^{-8}\right]$ does not change the computed values of $\rho_{\text {ins }}$ to six digits. It is not desirable to select $C_{1}$ too small (i.e. $<10^{-14}$ with double precision arithemtic) since numerical roundoff problems arise in this case [23].

In addition to the parameters in Table 7.1, the surrender charge imposed when a withdrawal occurs (denoted as $\hat{\gamma}(t)$ in equation $(2.10)$ ) is defined as in [35]:

$$
\gamma(t)= \begin{cases}0.08-0.01\lceil t\rceil & t \leq 7 \text { years } \\ 0.00 & t>7 \text { years }\end{cases}
$$

where $\lceil\cdot\rceil$ represents the ceiling function.

To determine the accuracy level that can be attained, we carry out a convergence analysis when pricing the GMDB guarantee. Table 7.2 holds the cost of the GMDB guarantee assuming $\omega=\$ 80$ for different refinement levels when the parameters in Table 7.1 are used. Note that we have set $\rho_{\text {ins }}=0.008$ for the time being. The top section of Table 7.2 contains the values obtained when fully implicit timestepping is used while the bottom panel presents the values recovered when Crank-Nicolson timestepping is used. Constant timesteps are taken for both fully implicit and Crank-Nicolson timestepping and the initial timestep is $\Delta \tau=0.05$ years on the coarsest grid. To eliminate oscillations in the final Crank-Nicolson solution, two fully implicit timesteps are taken at the start of the solution process [39]. Note that Crank-Nicolson is not monotone, and hence is not guaranteed to converge to the viscosity solution.

We see that the results for the highest refinement level in Table 7.2 provide an acceptable level of accuracy. However, results from higher refinement levels would be required to establish a definite conclusion about the convergence rate of the numerical scheme with both timestepping methods considered. Clearly the results in Table 7.2 show that the convergence has not settled down to the asymptotic rate. Results from higher refinement levels were not generated due to the prohibitive running time for such large problems. Nonetheless, since our interest lies in determining the fair insurance fee associated with the contract, the results in Table 7.2 provide adequate accuracy for practical purposes. 


\begin{tabular}{c|ccc|ccc}
\hline \multicolumn{7}{c}{ Cost of a GMDB guarantee } \\
\hline $\begin{array}{c}\text { Refinement } \\
\text { Level }\end{array}$ & $S$ & $B$ & $D$ & Option Value & Difference & Ratio \\
\hline \multicolumn{7}{c}{ Fully Implicit } \\
\hline \hline 0 & 36 & 36 & 21 & 1.653844 & n.a. & n.a. \\
1 & 71 & 71 & 41 & 1.728004 & 0.074161 & n.a. \\
2 & 141 & 141 & 81 & 1.752456 & 0.024452 & 3.03 \\
\hline \hline \multicolumn{7}{c}{ Crank-Nicolson } \\
\hline \hline 0 & 36 & 36 & 21 & 1.711003 & n.a. & n.a. \\
1 & 71 & 71 & 41 & 1.761588 & 0.050585 & n.a. \\
2 & 141 & 141 & 81 & 1.769926 & 0.008338 & 6.07 \\
\hline
\end{tabular}

Table 7.2: Cost of the GMDB guarantee when the owner is assumed to be a male of 50 years old at the time of purchase, $\omega=\$ 80$ and $\rho_{i n s}=0.008$. Other contract parameters are presented in Table 7.1. Nodes - $B$ indicates the maximum number of nodes in the $B$ direction (i.e. when $D=0$ ). The initial timestep is $\Delta \tau=0.05$ years on the coarsest grid.

\begin{tabular}{c|ccc|c}
\hline \multicolumn{5}{c}{ Fair Insurance Fee for GMDB Guarantee } \\
\hline Refinement & \multicolumn{3}{|c|}{ Nodes } & Insurance \\
Level & $S$ & $B$ & $D$ & Fee $\left(\rho_{\text {ins }}\right)$ \\
\hline 0 & 36 & 36 & 21 & 0.009255 \\
1 & 71 & 71 & 41 & 0.009225 \\
2 & 141 & 141 & 81 & 0.009216 \\
\hline
\end{tabular}

Table 7.3: Fair insurance fee $\left(\rho_{\text {ins }}\right)$ for a GMDB guarantee for different grid refinement levels when the owner is assumed to be a male of 50 years old at the time of purchase, $\omega=\$ 80$. Crank-Nicolson timestepping is used and the initial timestep is $\Delta \tau=0.05$ years on the coarsest grid. Other contract parameters are presented in Table 7.1. Nodes - $B$ indicates the maximum number of nodes in the $B$ direction (i.e. when $D=0$ ).

Table 7.3 presents the convergence of the fair risk charge obtained when we use Crank-Nicolson timestepping. As before we assume that the owner is male, 50 years old when the contract is purchased, and that $\omega=\$ 80$. Other contract parameters are set to the values presented in Table 7.1. Results for the highest refinement level in Table 7.3 suggest that the no-arbitrage fee is accurate to about $2 \times 10^{-5}$.

We also examined how the minimum deposit amount $(\omega)$ affects the fair insurance charge $\rho_{\text {ins }}$ obtained when solving equation (7.3). Table 7.4 presents the fair insurance charge for the GMDB clause with annual ratchet events when the minimum deposit $\omega$ ranges from $\$ 10$ to $\$ 90$. For comparison purposes, we also include the fair insurance charge for the GMDB clause when no withdrawals or contract lapsing are allowed. The results for both male and female owners are presented in Table 7.4. Other parameter values are specified in Table 7.1. In observing the results contained in Table 7.4, we see that the minimum deposit amount $\omega$ significantly impacts the fair insurance charge for the GMDB clause. Intuitively, as $\omega$ decreases, larger withdrawals can occur which is more detrimental to the issuing company and, as such, results in a higher insurance charge. The results in Table 7.4 show that the withdrawal feature is very valuable. 


\begin{tabular}{c|cccccc|c}
\hline & \multicolumn{6}{|c|}{ Minimal Deposit $\omega$} & No withdrawal \\
Owner & $\$ 90$ & $\$ 80$ & $\$ 60$ & $\$ 40$ & $\$ 20$ & $\$ 10$ & or lapsing \\
\hline \hline Male & 0.0090 & 0.0092 & 0.0097 & 0.0106 & 0.0123 & 0.0137 & 0.0077 \\
\hline Female & 0.0068 & 0.0069 & 0.0074 & 0.0081 & 0.0096 & 0.0108 & 0.0053 \\
\hline
\end{tabular}

Table 7.4: Fair insurance charge $\left(\rho_{\text {ins }}\right)$ for contracts containing a GMDB clause with annual ratchet events as a function of the minimal deposit amount $(\omega)$. Contract owners are assumed to be 50 years old at the time of purchase. The parameters in Table 7.1 are used in the pricing process.

\begin{tabular}{c|ccccc}
\hline & \multicolumn{5}{|c}{ Ratchet Interval } \\
Owner & 0.5 year & 1 year & 2 years & 5 years & 10 years \\
\hline Male & 0.0137 & 0.0123 & 0.0105 & 0.0080 & 0.0059 \\
Female & 0.0107 & 0.0095 & 0.0082 & 0.0062 & 0.0046 \\
\hline
\end{tabular}

Table 7.5: Fair insurance charge $\left(\rho_{\text {ins }}\right)$ for a GMDB guarantee with different ratchet intervals ranging from 0.5 to 10 years. The owner is assumed to be 50 years old at the time of purchase and $\omega=\$ 20$. Other contract parameters used when solving equation (7.3) are presented in Table 7.1.

Table 7.4 also demonstrates the impact of the gender of the contract owner on the required insurance charge. Since female owners generally live longer than their male counterparts, a lower insurance fee is required. As shown in Table 7.4, this can be observed for different values of $\omega$, as well as when the GMDB does not allow withdrawals or lapsing.

In [33], the authors state that certain contracts with a GMDB clause include longer time intervals between ratchet dates such as 2 or 5 years. As such, numerical results for pricing GMDB contracts with $\omega=\$ 20$ for different ratchet intervals ranging from 6 months to 10 years are presented in Table 7.5. Note that the parameter values presented in Table 7.1 are used and that the owner is assumed to be 50 years of age when the contract is purchased. The results of Table 7.5 demonstrate that a lower insurance charge is imposed by the issuer as the ratchet interval is increased. With fewer ratchet events during the contract lifetime, the death benefit exposure of the issuing company is generally reduced resulting in a lower insurance fee. This relation is observed for both male and female owners. Clearly, modifying the ratchet interval also significantly impacts the fair insurance charge associated with the GMDB clause. It would appear that both the withdrawal and ratchet features are very valuable when included in a GMDB contract.

\subsection{Numerical Results with Regime Switching}

We now consider results from numerical experiments where regime-switching is added to the pricing model, as described in Section 3. In accordance with the calibration carried out in [2], we assume that there are three economic regimes which we denote as $e_{1}, e_{2}$ and $e_{3}$. In [2], the authors assume that the underlying is in one of three regimes of Brownian volatility and calibrate this model to an existing volatility smile. Therefore, we will determine the fair insurance charge $\rho_{\text {ins }}$ that satisfies:

$$
V\left(\rho_{\text {ins }} ; S=\$ 100, B=\$ 100, D=\$ 100, E=e_{1}, \tau=40 \text { years }\right)=0 .
$$

The data for all three states, $e_{1}, e_{2}$ and $e_{3}$, is presented in Table 7.6 and is taken from [2]. Table 7.6 also includes additional information about contract parameters and details on the grid construction used when solving equation (7.5) for different values of $\omega$. We have verified that our choice for $B_{\max }$, 


\begin{tabular}{lc}
\hline \multicolumn{2}{c}{ State Information - $e_{1}$} \\
\hline$\sigma_{1}$ - Volatility & 0.0955 \\
Jump sizes: & $J^{1 \rightarrow 2}=0.9095 ; J^{1 \rightarrow 3}=1.0279$ \\
Jump intensities: & $\lambda^{1 \rightarrow 2}=0.2405 ; \lambda^{1 \rightarrow 3}=3.3208$ \\
\hline \multicolumn{2}{c}{ State Information - $e_{2}$} \\
\hline$\sigma_{2}$ - Volatility & 0.0644 \\
Jump sizes: & $J^{2 \rightarrow 1}=1.2502 ; J^{2 \rightarrow 3}=1.6512$ \\
Jump intensities: & $\lambda^{2 \rightarrow 1}=1.1279 ; \lambda^{2 \rightarrow 3}=0.0729$ \\
\hline \multicolumn{2}{c}{ State Information - $e_{3}$} \\
\hline$\sigma_{3}$ - Volatility $\quad 0.0241$ \\
Jump sizes: Contract Information \\
Jump intensities: & $\lambda^{3 \rightarrow 1}=0.9693 ; J^{3 \rightarrow 2}=0.7732$ \\
\hline \multicolumn{2}{c}{0.06} \\
\hline$r$ - Interest rate & 0.015 \\
$\rho_{\text {man }}$ - Management fees & 1 year \\
Ratchet interval & 30 years \\
Last Ratchet Date & Grid Construction \\
T - Contract maturity & 40 years \\
\hline \multicolumn{2}{c}{$\$ 100$} \\
\hline $\mathcal{D}_{0}$ - Initial deposit & $\$ 3.6 \times 10^{7}$ \\
$S_{\text {max }}$ - Grid parameter & $\$ 60000$ \\
$B_{\text {max }}$ - Grid parameter & \\
\hline
\end{tabular}

Table 7.6: Parameter values used when pricing GMDB contracts with regime-switching. Jump sizes and intensities taken from [2].

and consequently $S_{\max }$, still provides a minimum of 5 digits of accuracy in the numerical results obtained. We set the small fixed cost to $c=1 \times 10^{-10}$ to ensure accuracy of at least 6 digits in the numerical results obtained.

Table 7.7 holds the fair insurance fee for a GMDB guarantee with regime-switching assuming $\omega=\$ 80$ for different grid refinement levels. We further assume that the contract owner is a male of 50 years of age when the contract is purchased. Additional contract parameters are presented in Table 7.6 and constant timesteps are used with fully implicit timestepping. The initial timestep is $\Delta \tau=0.05$ years on the coarsest grid. Due to the high dimensionality of the pricing problem considered, the coarsest grid in the $D$ direction is limited to 11 nodes and results from only 2 refinement levels were obtained. We estimate that the results are correct to within $2 \times 10^{-4}$ when using a grid refinement of 2 . While results from higher refinement levels would be necessary to establish a more definite convergence analysis, problem size and running time would be unmanageable. We remind the reader that the regime switching HJB problem is four dimensional. Note that typically, one obtains convergence estimates for nonlinear HJB equations which are of the form $O\left(h^{\rho}\right)$ where $h$ is the discretization parameter. Estimates of $\rho$ vary from $1 / 27$ to $1 / 2$ depending on assumptions about regularity of the solution and the PDE coefficients. See [5] for an overview of recent work along these lines.

Table 7.8 holds the fair insurance charge associated with the GMDB guarantee as a function of $\omega$ assuming the economy is in state $e_{1}$. Based on previous comments, the results in Table 7.8 are 


\begin{tabular}{c|ccc|c}
\hline \multicolumn{5}{c}{ Fair Insurance Fee for GMDB Guarantee } \\
with Regime-Switching \\
\hline $\begin{array}{c}\text { Refinement } \\
\text { Level }\end{array}$ & $S$ & $B$ & $D$ & Fodes $\left(\rho_{\text {ins }}\right)$ \\
\hline 0 & 119 & 36 & 11 & 0.006286 \\
1 & 237 & 71 & 21 & 0.006085 \\
2 & 473 & 141 & 41 & 0.005931 \\
\hline
\end{tabular}

Table 7.7: Fair insurance fee $\left(\rho_{\text {ins }}\right)$ for a GMDB guarantee with regime-switching for different grid refinement levels. The owner is assumed to be a male of 50 years old at the time of purchase and $\omega=\$ 80$. Fully implicit timestepping is used and the initial timestep is $\Delta \tau=0.05$ years on the coarsest grid. Other contract parameters are presented in Table 7.1. Nodes - B indicates the maximum number of nodes in the $B$ direction (i.e. when $D=0$ ).

\begin{tabular}{c|cccccc|c}
\hline & \multicolumn{5}{|c|}{ Minimal Deposit $\omega$} & No withdrawal \\
Owner & $\$ 90$ & $\$ 80$ & $\$ 60$ & $\$ 40$ & $\$ 20$ & $\$ 10$ & or lapsing \\
\hline \hline Male & 0.0058 & 0.0059 & 0.0063 & 0.0070 & 0.0082 & 0.0091 & 0.0049 \\
Female & 0.0044 & 0.0045 & 0.0049 & 0.0054 & 0.0065 & 0.0073 & 0.0034 \\
\hline
\end{tabular}

Table 7.8: Fair insurance charge $\left(\rho_{\text {ins }}\right)$ for contracts containing a GMDB clause with annual ratchet events as a function of the minimal deposit amount $(\omega)$ assuming the economy is in regime $e_{1}$. Contract owners are assumed to be 50 years old at the time of purchase. The parameters in Table 7.6 are used in the pricing process.

obtained with a grid refinement level 2. Note that the owner is once again assumed to be 50 years of age when the contract is purchased. Other contract parameters used during the pricing process are presented in Table 7.6. For comparison purposes, the fair insurance charge associated with the GMDB guarantee when no withdrawal or lapsing is allowed is included in the last column of Table 7.8. As noted previously in Section 7.2, decreasing the minimum deposit amount $\omega$ increases the insurance fee charged by the issuing company. For example, setting $\omega=\$ 10$ when the contract owner is a man, requires a fee close to twice as large as that charged when no partial withdrawals are allowed. Notice that this remark applies equally to both male and female contract owners. In addition, the gender of the contract owner still affects the fair insurance charge for a given value of $\omega$. Assuming $\omega=\$ 40$, the fair insurance charge for the GMDB guarantee when owned by a woman is about $25 \%$ less than what is charged for a male contract owner. Thus, even when more realistic assumptions are made regarding the state of the economy, we see that both the gender of the contract owner and the value of $\omega$ have a significant impact on the fair insurance fee for the GMDB guarantee.

The results presented in Table 7.8 are significantly different from those included in previous work on the topic such as [33]. In [33], the authors consider a GMDB contract with continuous ratchet events, no partial withdrawals and a shorter maturity period, resulting in much lower insurance fees than those presented in Table 7.8. Thus, Table 7.8 clearly demonstrates that higher fees are required for GMDB contracts with a partial withdrawal feature in a regime-switching context. 


\section{Conclusion}

Increasingly popular in both the United States and the United Kingdom, variable annuity contracts include many different features. Focusing on contracts with a guaranteed minimum death benefit (GMDB) clause, we characterize the pricing problem as an impulse control problem. A pricing model based on partial differential equations was developed to determine the fair or no-arbitrage insurance charge for contracts with a GMDB clause. Regime-switching is also included in the pricing model due to the longer maturity of the contract considered. A numerical scheme was given which was shown to converge to the viscosity solution away from the ratchet dates. Based on results from numerical experiments, we have also shown that a much higher insurance charge is required when partial withdrawals are added to the GMDB guarantee. Previous work in the area [33] which ignores the possibility of partial withdrawals results in lower insurance fees. Inaccurate pricing and hedging of variable annuities has caused many insurance companies to take massive mark-to-market writedowns.

The most costly aspect of the computation of the guarantee involves the linear search for finding the optimal withdrawal. Further work will focus on techniques for speeding up this computation. While we have shown that our procedure converges to the viscosity solution, we are not able to determine the rate of convergence. It is interesting to note that this popular contract results in a complex optimal control problem which puts us close to the boundaries of the computing resources which would typically be available in an insurance company.

\section{A Derivation of the GMDB Guarantee Equation}

We summarize the approach used in $[42,43]$ to derive the GMDB guarantee equation $(2.5)$. Let $S$ be the amount in the investor's account (a mutual fund), so that $S$ follows the process

$$
d S=\left(\mu-\rho_{\text {total }}\right) S d t+\sigma S d Z,
$$

where $\mu$ is the drift under the real world measure. Recall that

$$
\rho_{\text {total }}=\rho_{\text {man }}+\rho_{\text {ins }}
$$

where $\rho_{\text {man }}$ are the management fees for the underlying mutual fund, and $\rho_{\text {ins }}$ are the fees allocated for funding the guarantee. More discussion of this typical fee splitting can be found in [43]. We suppose that the guarantee is offered on a mutual fund which tracks an index, so that it can be hedged without basis risk using index participation units. The index units $\hat{S}$ follow the process

$$
d \hat{S}=\mu \hat{S} d t+\hat{S} \sigma d Z
$$

We further assume that it is not possible to short the mutual fund, so that the obvious arbitrage opportunity cannot be exploited.

Now, consider the writer of the GMDB guarantee, with no-arbitrage value $V(S, B, D, t)$. The writer sets up the hedging portfolio

$$
\Pi(S, \hat{S}, t)=-V+x \hat{S}
$$

where $x$ is the number of units of the index $\hat{S}$. 
Over the time interval $t \rightarrow t+d t$, between withdrawal dates,

$$
\begin{aligned}
d \Pi= & -\left[\left(V_{t}+\left(\mu-\rho_{\text {total }}\right) S V_{S}+\frac{1}{2} \sigma^{2} S^{2} V_{S S}\right) d t+\sigma S V_{S} d Z\right] \\
& +x[\mu \hat{S} d t+\sigma \hat{S} d Z]+\mathcal{R}(t) \rho_{\text {ins }} S d t-\mathcal{M}(t) f d t,
\end{aligned}
$$

where the term $\mathcal{R}(t) \rho_{\text {ins }} S d t$ represents the GMDB fees collected from the investors remaining in the guarantee at time $t$, and the term $\mathcal{M}(t) f d t$ represents the death benefits paid out by the hedger. Let

$$
x=\frac{S}{\hat{S}} V_{S}
$$

so that equation (A.5) becomes

$$
d \Pi=-\left[\left(V_{t}-\rho_{\mathrm{total}} S V_{S}+\frac{1}{2} \sigma^{2} S^{2} V_{S S}\right) d t\right]+\mathcal{R}(t) \rho_{\text {ins }} S d t-\mathcal{M}(t) f d t .
$$

Let $r$ be the risk free rate. Then setting $d \Pi=r \Pi d t$ (since the portfolio is now riskless) gives

$$
V_{t}+\frac{1}{2} \sigma^{2} S^{2} V_{S S}+\left(r-\rho_{\text {total }}\right) S V_{S}-r V-\mathcal{R}(t) \rho_{\text {ins }} S+\mathcal{M}(t) f=0,
$$

which is equation $(2.5)$.

\section{B Death Benefits for GMDB Problem}

In this section we give some details on determining the death benefit exposure for the issuer of a GMDB contract. We will assume that the economy state is constant for this section and that $V=V(S, B, D, t)$ denotes the cost of the GMDB contract from the issuer's point of view.

When a GMDB contract is issued $(t=0)$, the death benefit is set to the initial deposit $\mathcal{D}_{0}$ made by the policy owner, that is, $B=\mathcal{D}_{0}$ at $t=0$. The death benefit can then be reset at each ratchet date to the maximum of the current investment account value or the current benefit level. Generally, ratchet events only occur during the accumulation phase of the contract and the last ratchet date is typically scheduled at the end of the policy year when the owner turns 80 years old [37]. If $t_{o}$ denotes a rachet date and $t_{o}^{-}$and $t_{o}^{+}$are times just before and after $t_{o}$ then standard no-arbitrage arguments give

$$
V\left(S, B^{+}, D, t_{o}^{+}\right)=V\left(S, B^{-}, D, t_{o}^{-}\right)
$$

where $B^{+}=\max \left(B^{-}, S\right)$.

Should the policy owner pass away prior to the expiry of the GMDB contract, the death benefit is exercised and the beneficiary receives the greater of the current benefit level or the current investment account value. Consequently, the issuing company is liable for any excess payment when the current death benefit is higher than the investment account value.

When the holder of the contract makes a partial or full withdrawal (lapsing), a surrender charge, denoted as $\gamma(t)$, is imposed. When the death benefit is exercised, the owner's estate does not pay a surrender charge. However, the issuer may have to pay a surrender charge to the re-seller [37]. In this paper, we consider the value of the guarantee from the issuer's perspective. To be concrete, we 
can think of the issuer of the guarantee as a re-insurer, and the re-seller as an insurance company selling the guarantee to retail customers. We assume that the surrender charge is calculated as a percentage of the current deposit level $D$ [37]. Generally, the surrender charge is highest at the start of the contract and decreases annually. After the initial $t_{s}$ years of the contract, the surrender charge disappears: $\gamma(t)=0$ when $t>t_{s}$ years. Typically, $t_{s}=7$ years. Hence, the death benefit exposure of the issuer, denoted by $f=f(S, B, D, t)$, is defined as:

$$
f(S, B, D, t)=\max (B-S, 0)+\gamma(t) D .
$$

\section{Partial Withdrawal Features}

In this section we give the details involved in allowing a partial withdrawal feature to be included in a GMDB contract, and give an intuitive derivation of equation (2.8). For a more detailed description of impluse control problems in finance, we refer the reader to [31].

The partial withdrawal feature enables the contract owner to withdraw any cash amount up to the current account value $S$. However, to keep the policy active, a minimal deposit amount must remain in the investment account. We denote the partial withdrawal amount as $W \in[0, S-\omega]$, where $\omega$ is the minimal deposit amount. For each partial withdrawal, a surrender charge, denoted by $\gamma(t)$ and calculated as a percentage of $W$, is imposed. The surrender charge $\gamma(t)$ is also applied when the owner chooses to lapse his policy. Recall that when an investor decides to lapse his policy, the investment account is liquidated and the GMDB policy cancelled. In this case, the surrender charge is a percentage of the investment account value $S$.

While we determine the no-arbitrage insurance charge for the GMDB guarantee, for explanatory purposes, it is useful to first consider the effect of partial withdrawals on the entire GMDB contract (investment account plus guarantee) and determine the appropriate withdrawal constraint. The withdrawal constraint for the entire GMDB contract is then used as a tool to derive the withdrawal constraint for the GMDB guarantee.

Let $\mathcal{V}=\mathcal{V}(S, B, D, t)$ represent the value of the entire GMDB contract (investment account plus guarantee). Assuming optimal behavior and ignoring mortality effects for the moment, the policy owner will maximize his return and choose $W$ such that:

$$
W=\underset{W^{\prime} \in[0, S-\omega]}{\operatorname{argmax}}\left((1-\gamma(t)) W^{\prime}+\mathcal{V}\left(S-W^{\prime}, \max \left(B-W^{\prime}, 0\right), \max \left(D-W^{\prime}, 0\right), t\right)\right) .
$$

Taking into consideration the option to lapse, the value of the total GMDB contract satisfies (after optimal withdrawal or lapsing):

$$
\mathcal{V}=\max \left((1-\gamma(t)) S, \max _{W \in[0, S-\omega]}((1-\gamma(t)) W+\mathcal{V}(S-W, \max (B-W, 0), \max (D-W, 0), t))\right) .
$$

While we have assumed in equation (C.2) that the contract owner will lapse whenever it is optimal to do so, alternate assumptions could be made whereby the contract owner would lapse at a predetermined rate. See $[42,43]$ for more details on modeling investor lapsing.

Our goal is to determine the value of the GMDB guarantee, so we need derive the equivalent withdrawal constraint from the issuer's perspective. We are looking to value the GMDB guarantee 
in an aggregate sense by assuming that contracts are sold to a given population. As such, the mortality/survival function defined in equation (2.6) must be taken into consideration when determining the withdrawal constraint. More precisely, we redefine $\mathcal{V}(S, B, D, t)$ as the value of the whole contract to the issuer which can be written as: $\mathcal{V}(S, B, D, t)=V(S, B, D, t)+\mathcal{R}(t) S$. Notice that only the investment account is affected by the survival probability since investor mortality is already included in the differential equation for $V(S, B, D, t)$ presented as (2.5). Since only those owners that are alive can conduct a withdrawal or choose to lapse, the cash flows associated with both actions will also be scaled by the survival probability.

Integrating our cash flow assumption into equation (C.2), we obtain

$$
\begin{aligned}
& \mathcal{V}=\max (\mathcal{R}(t)(1-\gamma(t)) S \\
&\left.\max _{W \in[0, S-\omega]}(\mathcal{R}(t)(1-\gamma(t)) W+\mathcal{V}(S-W, \max (B-W, 0), \max (D-W, 0), t))\right) \\
& \max (\mathcal{R}(t)(1-\gamma(t)) S \\
&\left.\max _{W \in[0, S-\omega]}(-\mathcal{R}(t) \gamma(t) W+V(S-W, \max (B-W, 0), \max (D-W, 0), t)+\mathcal{R}(t) S)\right),
\end{aligned}
$$

which, since $V(S, B, D, t)=\mathcal{V}(S, B, D, t)-\mathcal{R}(t) S$, gives

$$
V=\max \left(-\mathcal{R}(t) \gamma(t) S, \max _{W \in[0, S-\omega]}(V(S-W, \max (B-W, 0), \max (D-W, 0), t)-\mathcal{R}(t) \gamma(t) W)\right) .
$$

Thus, we can denote the withdrawal constraint by $\mathcal{A} V=\mathcal{A} V(S, B, D, t)$ with:

$\mathcal{A} V \equiv \max \left(-\mathcal{R}(t) \gamma(t) S, \max _{W \in[0, S-\omega]}(V(S-W, \max (B-W, 0), \max (D-W, 0), t)-\mathcal{R}(t) \gamma(t) W)-c\right)$, where equation
operator $\mathcal{L}$ as

$$
\mathcal{L} V=\frac{1}{2} \sigma^{2} S^{2} V_{S S}+\left(r-\rho_{\text {total }}\right) S V_{S}-r V
$$

then at all points in the soltion domain we have (from equation (2.5) )

$$
V_{\tau}-\mathcal{L} V+\mathcal{R}(\tau) \rho_{\text {ins }} S-\mathcal{M}(\tau) f \geq 0
$$

where equality holds if it is not optimal to withdraw. Since it must be optimal to either withdraw or not to withdraw, we have that

$$
\min \left(V_{\tau}-\mathcal{L} V+\mathcal{R}(\tau) \rho_{\text {ins }} S-\mathcal{M}(\tau) f, V-\mathcal{A} V\right)=0 .
$$

at all points in the solution domain. 


\section{Derivation of the Boundary Condition as $S \rightarrow \infty$}

To determine the boundary condition for equation (2.15) as $S \rightarrow \infty$, we make the common assumption that $V_{S S} \rightarrow 0$ [44], which implies:

$$
V \approx H(B, D, \tau) S+F(B, D, \tau)
$$

where $H(B, D, \tau)$ and $F(B, D, \tau)$ are independent of $S$. We further assume that $S$ is so large that $H(B, D, \tau) S \gg F(B, D, \tau)$, which leads to:

$$
V \approx H(B, D, \tau) S
$$

Equation (D.2) implies:

$$
V_{S} \approx H(B, D, \tau)
$$

and hence, we can rewrite the differential equation in (2.15) as:

$$
\begin{aligned}
H_{\tau}(B, D, \tau) S= & \left(r-\rho_{\text {total }}\right) H(B, D, \tau) S-r H(B, D, \tau) S-\mathcal{R}(\tau) \rho_{\text {ins }} S+\mathcal{M}(\tau) \max (B-S, 0) \\
& +\mathcal{M}(\tau) \gamma(\tau) D+\frac{1}{\epsilon} \max (\mathcal{A}(H(B, D, \tau) S)-H(B, D, \tau) S, 0)
\end{aligned}
$$

where

$$
\begin{aligned}
& \mathcal{A}(H(B, D, \tau) S)= \\
& \quad \max \left(-\mathcal{R}(\tau) \gamma(\tau) S, \max _{W \in[0, S-\omega]}(H(\max (B-W, 0), \max (D-W, 0), \tau)(S-W)-\mathcal{R}(\tau) \gamma(\tau) W)-c\right) .
\end{aligned}
$$

Since $B \ll S_{\max }$ and $W \leq \mathcal{D}_{0} \ll S_{\max }$, we can simplify equation (D.4) as:

$$
\begin{aligned}
& H_{\tau}(B, D, \tau) S \approx \\
& \quad-\rho_{\text {total }} H(B, D, \tau) S-\mathcal{R}(\tau) \rho_{\text {ins }} S+\frac{1}{\epsilon} \max (\mathcal{A}(H(B, D, \tau) S)-H(B, D, \tau) S, 0) .
\end{aligned}
$$

As a result, we obtain the following approximation to equation (D.4):

$$
V_{\tau}=-\rho_{\text {total }} V-\mathcal{R}(\tau) \rho_{\text {ins }} S+\frac{1}{\epsilon} \max (\mathcal{A} V-V, 0) ; S=S_{\text {max }} .
$$

A similar argument gives the boundary condition for large $S$ when regime switching is used.

\section{E Discretization}

The regime-switching partial differential equation presented in (3.8) can be approximated by replacing derivatives by finite difference approximations. Recall that the discrete version of equation (3.8) can be written as in equation (5.3) (assuming fully implicit timestepping).

The choice of discretization for the derivative terms in equation (3.8) will determine the value of both $\alpha_{i, j, m}$ and $\beta_{i, j, m}$. For example, choosing the higher order central difference scheme leads to 
if $\alpha_{i, j, m, \text { central }} \geq 0$ and $\beta_{i, j, m, \text { central }} \geq 0$ then

$\alpha_{i, j, m}=\alpha_{i, j, m, \text { central }}$

$\beta_{i, j, m}=\beta_{i, j, m, \text { central }}$

else if $\beta_{i, j, m \text {,forward }} \geq 0$ then

$\alpha_{i, j, m}=\alpha_{i, j, m, \text { forward }}$

$\beta_{i, j, m}=\beta_{i, j, m, \text { forward }}$

else

$\alpha_{i, j, m}=\alpha_{i, j, m, \text { backward }}$

$\beta_{i, j, m}=\beta_{i, j, m, \text { backward }}$

end if

Algorithm E.1: Coefficient Discretization

681

the following values of $\alpha_{i, j, m}$ and $\beta_{i, j, m}$ :

$$
\begin{aligned}
\alpha_{i, j, m, \text { central }} & =\frac{\left(\sigma_{m} S_{i}^{j}\right)^{2}}{\left(S_{i}^{j}-S_{i-1}^{j}\right)\left(S_{i+1}^{j}-S_{i-1}^{j}\right)}-\frac{S_{i}^{j}\left(r-\rho_{\text {total }}-\sum_{l=1 ; l \neq m}^{M} \lambda^{m \rightarrow l}\left(J_{i, j}^{m \rightarrow l}-1\right)\right)}{S_{i+1}^{j}-S_{i-1}^{j}}, \\
\beta_{i, j, m, \text { central }} & =\frac{\left(\sigma_{m} S_{i}^{j}\right)^{2}}{\left(S_{i+1}^{j}-S_{i}^{j}\right)\left(S_{i+1}^{j}-S_{i-1}^{j}\right)}+\frac{S_{i}^{j}\left(r-\rho_{\text {total }}-\sum_{l=1 ; l \neq m}^{M} \lambda^{m \rightarrow l}\left(J_{i, j}^{m \rightarrow l}-1\right)\right)}{S_{i+1}^{j}-S_{i-1}^{j}},
\end{aligned}
$$

where $J_{i, j}^{m \rightarrow l}=J^{m \rightarrow l}\left(S_{i}^{j}\right)$. However, to produce a positive coefficient method, it is preferable to choose other discretization techniques at the problem nodes such as forward or backward differences. Forward differences produces:

$$
\begin{aligned}
\alpha_{i, j, m, \text { forward }} & =\frac{\left(\sigma_{m} S_{i}^{j}\right)^{2}}{\left(S_{i}^{j}-S_{i-1}^{j}\right)\left(S_{i+1}^{j}-S_{i-1}^{j}\right)}, \\
\beta_{i, j, m, \text { forward }} & =\frac{\left(\sigma_{m} S_{i}^{j}\right)^{2}}{\left(S_{i+1}^{j}-S_{i}^{j}\right)\left(S_{i+1}^{j}-S_{i-1}^{j}\right)}+\frac{S_{i}^{j}\left(r-\rho_{\text {total }}-\sum_{l=1 ; l \neq m}^{M} \lambda^{m \rightarrow l}\left(J_{i, j}^{m \rightarrow l}-1\right)\right)}{S_{i+1}^{j}-S_{i}^{j}},
\end{aligned}
$$

while backward differences delivers:

$$
\begin{aligned}
\alpha_{i, j, m, \text { backward }} & =\frac{\left(\sigma_{m} S_{i}^{j}\right)^{2}}{\left(S_{i}^{j}-S_{i-1}^{j}\right)\left(S_{i+1}^{j}-S_{i-1}^{j}\right)}-\frac{S_{i}^{j}\left(r-\rho_{\text {total }}-\sum_{l=1 ; l \neq m}^{M} \lambda^{m \rightarrow l}\left(J_{i, j}^{m \rightarrow l}-1\right)\right)}{S_{i+1}^{j}-S_{i}^{j}}, \\
\beta_{i, j, m, \text { backward }} & =\frac{\left(\sigma_{m} S_{i}^{j}\right)^{2}}{\left(S_{i+1}^{j}-S_{i}^{j}\right)\left(S_{i+1}^{j}-S_{i-1}^{j}\right)} .
\end{aligned}
$$

Algorithmically, the decision between a central or forward discretization at each node is made based on the criteria presented in Algorithm E.1. Note that Algorithm E.1 guarantees that both $\alpha_{i, j, m}$ and $\beta_{i, j, m}$ are non-negative:

$$
\alpha_{i, j, m} \geq 0 ; \beta_{i, j, m} \geq 0 \quad \text { for all } i, j \text { and } m \text {. }
$$




\section{F Proofs of Stability and Consistency of Discretization}

In this section we give proofs of both stability and consistency of our discretization in order to complete our theoretical analysis of the previous section. We note that such proofs are usually loosely presented without any details. However the details are often subtle and in order to ensure correctness we give the complete proofs.

\section{F.1 Proof of Theorem 6.7}

In this subsection, we show that the discrete GMDB cost $V_{i, j, k, m}^{n+1}$ is bounded. Before proving Theorem 6.7 , we prove some utility lemmas. We define the vector $V^{n+1}$ as:

$$
V^{n+1}=\left[\begin{array}{c}
V_{1}^{n+1} \\
V_{2}^{n+1} \\
\vdots \\
V_{M}^{n+1}
\end{array}\right],
$$

where $V_{m}^{n+1}$ is defined in equation $(5.12)$ and the $\kappa^{t h}$ entry of $V^{n+1}$ is denoted as $\left[V^{n+1}\right]_{i, j, k, m}$ where:

$$
\kappa=(i+1)+j\left(i_{\max }+1\right)+k\left(i_{\max }+1\right)\left(j_{\max }+1\right)+(m-1)\left(i_{\max }+1\right)\left(j_{\max }+1\right)\left(k_{\max }+1\right) .
$$

Let $\mathcal{P}^{n+1}$ be defined as:

$$
\begin{gathered}
{\left[\mathcal{P}^{n+1} \mathcal{Z}^{n+1}\right]_{i, j, k, m}=\left(1+\Delta \tau\left(\alpha_{i, j, m}+\beta_{i, j, m}+r+\sum_{\substack{l=1 \\
l \neq m}}^{M} \lambda^{m \rightarrow l}\right)\right) \mathcal{Z}_{i, j, k, m}^{n+1}-\Delta \tau \alpha_{i, j, m} \mathcal{Z}_{i-1, j, k, m}^{n+1}} \\
-\Delta \tau \beta_{i, j, m} \mathcal{Z}_{i+1, j, k, m}^{n+1}-\Delta \tau \sum_{\substack{l=1 \\
l \neq m}}^{M} \lambda^{m \rightarrow l} \mathcal{H}\left(J^{m \rightarrow l}\right)_{i} \mathcal{Z}_{j, k, l}^{n+1}
\end{gathered}
$$

when $i<i_{\max }$ and

$$
\begin{aligned}
{\left[\mathcal{P}^{n+1} \mathcal{Z}^{n+1}\right]_{i_{\max }, j, k, m}=} & \left(1+\Delta \tau\left(\rho_{\text {total }}+\sum_{\substack{l=1 \\
l \neq m}}^{M} \lambda^{m \rightarrow l} J_{i_{\max }}^{m \rightarrow l}\right)\right) \mathcal{Z}_{i_{\max }, j, k, m}^{n+1} \\
& -\Delta \tau \sum_{\substack{l=1 \\
l \neq m}}^{M} \lambda^{m \rightarrow l} J_{i_{\max }^{m \rightarrow l}}^{m} \mathcal{Z}_{i_{\max }, j, k, l}^{n+1}
\end{aligned}
$$

when $i=i_{\max }$. Also, let $\mathcal{Q}^{n+1}\left(V^{n+1}\right)$ be defined by:

$$
\begin{aligned}
{\left[\mathcal{Q}^{n+1}\left(V^{n+1}\right) \mathcal{Z}^{n+1}\right]_{i, j, k, m}=\left[\mathcal{P}^{n+1} \mathcal{Z}^{n+1}\right]_{i, j, k, m} } & +\frac{\Delta \tau \mu_{i, j, k, m}^{n+1}}{\epsilon} \mathcal{Z}_{i, j, k, m}^{n+1} \\
& -\frac{\Delta \tau \mu_{i, j, k, m}^{n+1}}{\epsilon}\left(1-a_{i, j, k, m}^{n+1}\right) \mathcal{I}\left(W_{i, j, k, m}^{n+1}\right)_{i, j, k} \mathcal{Z}_{m}^{n+1},
\end{aligned}
$$


valid for all $i$. Here, $\mu_{i, j, k, m}^{n+1}$ is defined in equation (5.6), $a_{i, j, k, m}^{n+1}$ is defined in equation (5.15) and the interpolation operators $\mathcal{H}\left(J^{m \rightarrow l}\right)_{i}$ and $\mathcal{I}\left(W_{i, j, k, m}^{n+1}\right)_{i, j, k}$ are defined in equations (5.9) and (5.13) respectively. The matrix $\mathcal{Q}^{n+1}\left(V^{n+1}\right)$ is the matrix of coefficients for all terms involving elements from $V^{n+1}$ in the discretization (5.3). Note that $\mathcal{Q}^{n+1}\left(V^{n+1}\right)$ is a function of the solution since the interpolation operators, the $\mu$ and $a$ values all depend on the solution.

It is useful to note the following property of the coefficient matrices $\mathcal{P}^{n+1}$ and $\mathcal{Q}^{n+1}\left(V^{n+1}\right)$.

Lemma F.1 (M-matrix). The matrices $\mathcal{P}^{n+1}$ and $\mathcal{Q}^{n+1}\left(V^{n+1}\right)$ as defined in equations (F.2),(F.3) and (F.4) are $M$-matrices for any $V^{n+1}$.

Proof. The diagonal entries in $\mathcal{P}^{n+1}$ are positive while the off-diagonal entries are negative or equal to zero. In addition, the row sum of the entries in both matrices are strictly positive for all rows. The above are also true for the matrix $\mathcal{Q}^{n+1}\left(V^{n+1}\right)$ for any $V^{n+1}$. Thus both $\mathcal{P}^{n+1}$ and $\mathcal{Q}^{n+1}\left(V^{n+1}\right)$ are M-matrices.

Remark F.2. We remark that an M-matrix has the important property that it is invertible with a positive inverse. In particular, for any vector $Z, \mathcal{P}^{n+1} Z \geq 0$ or $\mathcal{Q}^{n+1}\left(V^{n+1}\right) Z \geq 0$ implies that $Z \geq 0$.

Lemma F.3. The following are true.

(a) Let $\left[\mathcal{Z}^{n+1}\right]_{i, j, k, m}=C_{0}^{n+1} B_{\max }+C_{1}^{n+1} D_{\max }$ (with $C_{0}^{n+1}, C_{1}^{n+1}$ defined in (6.5)). Then:

$$
\mathcal{Q}^{n+1}\left(V^{n+1}\right) \mathcal{Z}^{n+1}>\mathcal{Z}^{n+1}
$$

for any $V^{n+1}$.

(b) Let $\left[\mathcal{Z}^{n+1}\right]_{i, j, k, m}=S_{i}^{j}$. Then ${ }^{3}$ :

$$
\mathcal{P}^{n+1} \mathcal{Z}^{n+1}=\left(1+\rho_{\text {total }} \Delta \tau\right) \mathcal{Z}^{n} .
$$

(c) Let $\mathcal{Z}$ solve the discrete equations (5.3). Then:

$$
\mathcal{Q}^{n+1}\left(\mathcal{Z}^{n+1}\right) \mathcal{Z}^{n+1}=\mathcal{Z}^{n}+\Delta \tau \text { Rest }^{n+1},
$$

where for all $i$ (since $f_{i_{\max }, j, k}=0$ )

$$
\begin{gathered}
\left.\operatorname{Rest}^{n+1}\right]_{i, j, k, m}=\mathcal{M}^{n+1} f_{i, j, k}-\mathcal{R}^{n+1} \rho_{i n s} S_{i}^{j}-\frac{\mu_{i, j, k, m}^{n+1}}{\epsilon}\left[a_{i, j, k, m}^{n+1} \mathcal{R}^{n+1} \gamma^{n+1} S_{i}^{j}\right. \\
\left.+\left(1-a_{i, j, k, m}^{n+1}\right)\left(\mathcal{R}^{n+1} \gamma^{n+1} W_{i, j, k, m}^{n+1}+c\right)\right]
\end{gathered}
$$

denotes the constant terms of the discretization.

\footnotetext{
${ }^{3}$ Note that this is trivially true at $S_{0}^{j}=0$.
} 
(d) Let $\mathcal{Z}$ solve the discrete equations (5.3). Then:

$$
\mathcal{P}^{n+1} \mathcal{Z}^{n+1}=\mathcal{Z}^{n}+\Delta \tau \operatorname{Rest}^{n+1},
$$

where for all $i$ (since $f_{i_{\max }, j, k}=0$ )

$$
\begin{aligned}
\text { Rest } \left.^{n+1}\right]_{i, j, k, m}= & \mathcal{M}^{n+1} f_{i, j, k}-\mathcal{R}^{n+1} \rho_{i n s} S_{i}^{j}+\frac{\mu_{i, j, k, m}^{n+1}}{\epsilon}\left[-a_{i, j, k, m}^{n+1} \mathcal{R}^{n+1} \gamma^{n+1} S_{i}^{j}\right. \\
& \left.+\left(1-a_{i, j, k, m}^{n+1}\right)\left(\mathcal{I}\left(W_{i, j, k, m}^{n+1}\right)_{i, j, k} \mathcal{Z}_{m}^{n+1}-\mathcal{R}^{n+1} \gamma^{n+1} W_{i, j, k, m}^{n+1}-c\right)-\mathcal{Z}_{i, j, k, m}^{n+1}\right] .
\end{aligned}
$$

Proof. Identity (a) follows by looking at the $i, j, k, m$ components of the matrix form of $\mathcal{P}$ and $\mathcal{Q}$. For example, when $i<i_{\max }$ we have

$$
\begin{aligned}
{\left[\mathcal{Q}^{n+1}\left(V^{n+1}\right) \mathcal{Z}^{n+1}\right]_{i, j, k, m} } & =\left(1+\Delta \tau\left(r+a_{i, j, k, m} \frac{\mu_{i, j, k, m}}{\epsilon}\right)\right)\left[\mathcal{Z}^{n+1}\right]_{i, j, k, m} \\
& >\left[\mathcal{Z}^{n+1}\right]_{i, j, k, m}
\end{aligned}
$$

with a similar inequality when $i=i_{\max }$. A similar argument holds for identity (b). Identities (c) and (d) follow directly from the definitions of $\mathcal{Q}$ and $\mathcal{P}$ and the discretization in (5.3).

We now present the proof of Theorem 6.7 .

Proof. (of Theorem 6.7)

Let $\mathcal{Z}^{n}$ be the vector defined by $\left[\mathcal{Z}^{n}\right]_{i, j, k, m}=S_{i}^{j}+V_{i, j, k, m}^{n}$ for all $i, j, k, m$. We will use induction to show that $\mathcal{Z}^{n} \geq 0$ for all $n$.

Notice that $\left[\mathcal{Z}^{0}\right]_{i, j, k, m}=S_{i}^{j}+V_{i, j, k, m}^{0}=S_{i}^{j} \geq 0$. Assume now that $n>0$ and that $\mathcal{Z}^{n} \geq 0$. Then, from Lemma F.3(b)(d) we have:

$$
\left[\mathcal{P}^{n+1} \mathcal{Z}^{n+1}\right]=\mathcal{Z}^{n}+\Delta \tau \mathcal{G}^{n+1}
$$

with (since $f_{i, j, k} \geq 0$ )

$$
\begin{aligned}
{\left[\mathcal{G}^{n+1}\right]_{i, j, k, m} \geq } & \left(\rho_{\text {total }}-\mathcal{R}^{n+1} \rho_{\text {ins }}\right) S_{i}^{j}+\frac{\mu_{i, j, k, m}^{n+1}}{\epsilon}\left[-a_{i, j, k, m}^{n+1} \mathcal{R}^{n+1} \gamma^{n+1} S_{i}^{j}\right. \\
& \left.+\left(1-a_{i, j, k, m}^{n+1}\right)\left(\mathcal{I}\left(W_{i, j, k, m}^{n+1}\right)_{i, j, k} V_{m}^{n+1}-\mathcal{R}^{n+1} \gamma^{n+1} W_{i, j, k, m}^{n+1}-c\right)-V_{i, j, k, m}^{n+1}\right] .(
\end{aligned}
$$

Note that $\rho_{\text {total }}-\mathcal{R}^{n+1} \rho_{\text {ins }} \geq 0$. Furthermore, notice that $\mu_{i, j, k, m}^{n+1}=1$ only when (see equation (5.6)):

$$
-a_{i, j, k, m}^{n+1} \mathcal{R}^{n+1} \gamma^{n+1} S_{i}^{j}+\left(1-a_{i, j, k, m}^{n+1}\right)\left(\mathcal{I}\left(W_{i, j, k, m}^{n+1}\right)_{i, j, k} V_{m}^{n+1}-\mathcal{R}^{n+1} \gamma^{n+1} W_{i, j, k, m}^{n+1}-c\right)-V_{i, j, k, m}^{n+1}>0
$$

and $\mu_{i, j, k, m}^{n+1}=0$ otherwise. Hence, equation (F.8) implies that $\left[\mathcal{G}^{n+1}\right]_{i, j, k, m} \geq 0$.

Since $\mathcal{Z}^{n} \geq 0$, we see that $\mathcal{P}^{n+1} \mathcal{Z}^{n+1} \geq 0$ and, since $\mathcal{P}^{n+1}$ is an M-matrix, $\mathcal{Z}^{n+1} \geq 0$. Thus, by induction $\mathcal{Z}^{n} \geq 0$ for all $n$, proving the first inequality of (6.4). 
Now let $\mathcal{Z}^{n}$ be the vector defined by $\left[\mathcal{Z}^{n}\right]_{i, j, k, m}=C_{0}^{n} B_{\max }+C_{1}^{n} D_{\max }$ for all $i, j, k, m$. We will prove the second inequality of (6.4) by using induction to show that $\mathcal{Z}^{n}-V^{n} \geq 0$ for all $n$. Since (see equation (6.5)):

$$
\left[\mathcal{Z}^{0}-V^{0}\right]_{i, j, k, m}=\Delta \tau \mathcal{M}^{0} B_{\max }+\Delta \tau \mathcal{M}^{0} \gamma^{0} D_{\max } \geq 0,
$$

the result is true for $n=0$. Assume that $n>0$ and that $\mathcal{Z}^{n}-V^{n} \geq 0$. From Lemma F.3(a) along with the definition of $C_{0}^{n}$ and $C_{1}^{n}$ (see equation (6.5)) we have:

$$
\mathcal{Q}^{n+1}\left(V^{n+1}\right) \mathcal{Z}^{n+1}>\mathcal{Z}^{n+1}=\mathcal{Z}^{n}+\Delta \tau\left[\mathcal{M}^{n+1} B_{\max }+\mathcal{M}^{n+1} \gamma^{n+1} D_{\max }\right] .
$$

Hence, using Lemma F.3(c) gives:

$$
\mathcal{Q}^{n+1}\left(V^{n+1}\right)\left(\mathcal{Z}^{n+1}-V^{n+1}\right)>\left(\mathcal{Z}^{n}-V^{n}\right)+\Delta \tau\left[\mathcal{M}^{n+1} B_{\max }+\mathcal{M}^{n+1} \gamma^{n+1} D_{\max }\right]-\Delta \tau \text { Rest }^{n+1},
$$

where the components of Rest ${ }^{n+1}$ are given in equation (F.5). Let

$$
\mathcal{G}=\left[\mathcal{M}^{n+1} B_{\max }+\mathcal{M}^{n+1} \gamma^{n+1} D_{\max }\right]-\text { Rest }^{n+1} .
$$

Then, for $i<i_{\max }$, and using:

$$
0 \leq f_{i, j, k}^{n+1}=\max \left(B_{j}^{k}-S_{i}^{j}, 0\right)+\gamma^{n+1} D_{k} \leq B_{\max }+\gamma^{n+1} D_{\max },
$$

we have:

$$
\begin{aligned}
{[\mathcal{G}]_{i, j, k, m}=} & \mathcal{M}^{n+1}\left(B_{\max }+\gamma^{n+1} D_{\max }-f_{i, j, k}\right)+\mathcal{R}^{n+1} \rho_{i n s} S_{i}^{j} \\
& \quad+\frac{\mu_{i, j, k, m}^{n+1}}{\epsilon}\left[a_{i, j, k, m}^{n+1} \mathcal{R}^{n+1} \gamma^{n+1} S_{i}^{j}+\left(1-a_{i, j, k, m}^{n+1}\right)\left(\mathcal{R}^{n+1} \gamma^{n+1} W_{i, j, k, m}^{n+1}+c\right)\right] \\
\geq & \mathcal{R}^{n+1} \rho_{i n s} S_{i}^{j} \\
& \quad+\frac{\mu_{i, j, k, m}^{n+1}}{\epsilon}\left[a_{i, j, k, m}^{n+1} \mathcal{R}^{n+1} \gamma^{n+1} S_{i}^{j}+\left(1-a_{i, j, k, m}^{n+1}\right)\left(\mathcal{R}^{n+1} \gamma^{n+1} W_{i, j, k, m}^{n+1}+c\right)\right] \\
\geq & 0,
\end{aligned}
$$

since there are only positive terms in the expression. This is also the case when $i=i_{\max }$. As before, $\mathcal{Z}^{n}-V^{n} \geq 0$ so that $\mathcal{Q}^{n+1}\left(V^{n+1}\right)\left(\mathcal{Z}^{n+1}-V^{n+1}\right) \geq 0$ and, since $\mathcal{Q}^{n+1}\left(V^{n+1}\right)$ is an Mmatrix, $\mathcal{Z}^{n+1}-V^{n+1} \geq 0$. Hence, by induction, $\mathcal{Z}^{n}-V^{n} \geq 0$ for all $n$.

Thus, we have shown that $V_{i, j, k, m}^{n+1}$ is bounded with:

$$
-S_{i}^{j} \leq V_{i, j, k, m}^{n+1} \leq \mathcal{C}_{0}^{n+1} B_{\max }+\mathcal{C}_{1}^{n+1} D_{\max } \text { for all } i, j, k, m, n .
$$

Note that the bound presented in equation (F.13) also holds immediately after each ratchet date $\tau_{o}^{u+}$. Recall that the value of the GMDB guarantee is updated on each ratchet date $\tau_{o}^{u}$ according to equation (4.8), which implies (for the continuous problem):

$$
V^{m}\left(S, B, D, e_{m}, \tau_{o}^{u+}\right)= \begin{cases}V^{m}\left(S, B, D, e_{m}, \tau_{o}^{u-}\right) & \text { if } S \leq B \\ V^{m}\left(S, S, D, e_{m}, \tau_{o}^{u-}\right) & \text { if } B<S \leq B_{\max } \\ V^{m}\left(S, B, D, e_{m}, \tau_{o}^{u-}\right) & \text { if } S>B_{\max }\end{cases}
$$

Equation (F.14) implies that the bound for $V_{i, j, k, m}^{n+1}$ presented in equation (F.13) remains applicable at times $\tau_{o}^{u+}$. 
Remark F.4 (Tighter Upper Bound). We note that it is possible to obtain the tighter bound

$$
-S_{i}^{j} \leq V_{i, j, k, m}^{n+1} \leq \mathcal{C}_{0}^{n+1} B_{\max }+\mathcal{C}_{1}^{n+1} D_{k} \text { for all } i, j, k, m, n .
$$

However, bound (6.4) is sufficient for our purposes.

\section{F.2 Proof of Theorem 6.13}

In this subsection, we show that the numerical scheme in equation (5.3) is consistent. Before proving Theorem 6.13 , we prove an important lemma.

Lemma F.5. For any smooth test function $\phi$ with bounded derivatives of all orders with respect to $S$ and $\tau$, with $x=\left(S_{i}^{j}, B_{j}^{k}, D_{k}, e_{m}, \tau^{n+1}\right)$, we have (see equation (6.13)):

$$
\hat{\mathcal{G}}\left(h, x, \phi_{i, j, k, m}^{n+1}+\xi, \phi_{i, j, k, m}^{n}+\xi,\left\{\phi_{a, p, u, l}^{n+1}+\xi\right\}\right)-F(\phi)_{i, j, k, m}^{n+1}=O(h)+\xi b(x),
$$

where $b(x)$ is a bounded function of $x$ with $|b(x)| \leq \max \left(r, \rho_{\text {total }}\right)$.

Proof. To prove Lemma F.5, we consider the truncation error for the differential operator $\mathcal{L}$ and the penalty term.

Let

$$
[\mathcal{L} \phi]_{i, j, k, m}^{n+1}
$$

represent the continuous operator $\mathcal{L}$ at node $\left(S_{i}^{j}, B_{j}^{k}, D_{k}, e_{m}, \tau^{n+1}\right)$, while the discrete version of the operator is denoted by:

$$
\left[\mathcal{L}^{h} \phi\right]_{i, j, k, m}^{n+1}
$$

Using Taylor series expansion, we have:

$$
\left[\mathcal{L}^{h}(\phi+\xi)\right]_{i, j, k, m}^{n+1}-[\mathcal{L} \phi]_{i, j, k, m}^{n+1}=-r \xi+O\left(\Delta S_{\max }^{j}\right),
$$

when computing $\mathcal{H}_{i} \phi_{j, k, l}^{n+1}$ using linear interpolation (see equation (5.9)).

Similarly, we assume that:

$$
[\mathcal{A} \phi]_{i, j, k, m}^{n+1}
$$

represents the continuous withdrawal constraint evaluated at node $\left(S_{i}^{j}, B_{j}^{k}, D_{k}, e_{m}, \tau^{n+1}\right)$, while the discrete version of the withdrawal constraint is denoted as:

$$
\left[\mathcal{A}^{h} \phi\right]_{i, j, k, m}^{n+1}
$$

Recall that the discrete withdrawal constraint is determined by linear search as in Algorithm 5.1.

The discretization error associated with the penalty term occurs when it is optimal for the owner to conduct a withdrawal, as opposed to lapsing his policy. Indeed, interpolation is required when calculating the penalty term when a withdrawal occurs, but not when the owner lapses (see equation (5.11)). Since the maximum of a linearly interpolated value is obtained at the nodes, the linear interpolation truncation error is $O\left(h^{2}\right)$ (noting Assumption 5.6). Taking the maximum of the linear interpolation function, as done in Algorithm 5.1, is also second order correct. Assuming twodimensional linear interpolation is used when calculating the withdrawal constraint as described 
in equation (5.13), the interpolation error will be $O\left(\Delta S_{\max }^{j} \Delta B_{\max }^{u}\right)$. Therefore, we obtain (from equation (5.16)):

$$
\left[\mathcal{A}^{h}(\phi+\xi)\right]_{i, j, k, m}^{n+1}-[\mathcal{A} \phi]_{i, j, k, m}^{n+1}=\xi+O\left(\Delta S_{\max }^{j} \Delta B_{\max }^{u}\right)+O\left(h^{2}\right)
$$

when it is optimal to withdraw and 0 when it is optimal to lapse.

Recall from equation (6.6) that the discrete scheme $\mathcal{G}\left(h, x, V_{i, j, k, m}^{n+1}, V_{i, j, k, m}^{n},\left\{V_{a, p, u, l}^{n+1}\right\}\right)$ is denoted as follows on interior nodes when $S_{i}^{j}<S_{\max }$ :

$\frac{\phi_{i, j, k, m}^{n+1}-\phi_{i, j, k, m}^{n}}{\Delta \tau}-\left[\mathcal{L}^{h} \phi\right]_{i, j, k, m}^{n+1}+\mathcal{R}^{n+1} \rho_{i n s} S_{i}^{j}-\mathcal{M}^{n+1} f_{i, j, k}^{n+1}-\frac{1}{\epsilon} \max \left(\left[\mathcal{A}^{h} \phi\right]_{i, j, k, m}^{n+1}-\phi_{i, j, k, m}^{n+1}, 0\right)=0$.

We re-formulate the penalized problem in equation (F.23) as:

$$
\begin{gathered}
\min \left[\frac{\phi_{i, j, k, m}^{n+1}-\phi_{i, j, k, m}^{n}}{\Delta \tau}-\left[\mathcal{L}^{h} \phi\right]_{i, j, k, m}^{n+1}+\mathcal{R}^{n+1} \rho_{i n s} S_{i}^{j}-\mathcal{M}^{n+1} f_{i, j, k}^{n+1}-\frac{1}{\epsilon}\left(\left[\mathcal{A}^{h} \phi\right]_{i, j, k, m}^{n+1}-\phi_{i, j, k, m}^{n+1}\right)\right. \\
\left.\frac{\phi_{i, j, k, m}^{n+1}-\phi_{i, j, k, m}^{n}}{\Delta \tau}-\left[\mathcal{L}^{h} \phi\right]_{i, j, k, m}^{n+1}+\mathcal{R}^{n+1} \rho_{i n s} S_{i}^{j}-\mathcal{M}^{n+1} f_{i, j, k}^{n+1}\right]=0
\end{gathered}
$$

Equation (F.24) implies that one of the following holds with equality:

$$
\begin{array}{r}
\frac{\phi_{i, j, k, m}^{n+1}-\phi_{i, j, k, m}^{n}}{\Delta \tau}-\left[\mathcal{L}^{h} \phi\right]_{i, j, k, m}^{n+1}+\mathcal{R}^{n+1} \rho_{i n s} S_{i}^{j}-\mathcal{M}^{n+1} f_{i, j, k}^{n+1}-\frac{1}{\epsilon}\left(\left[\mathcal{A}^{h} \phi\right]_{i, j, k, m}^{n+1}-\phi_{i, j, k, m}^{n+1}\right) \geq 0, \\
\frac{\phi_{i, j, k, m}^{n+1}-\phi_{i, j, k, m}^{n}}{\Delta \tau}-[\mathrm{F} .25) \\
\frac{\left.\mathcal{L}^{h} \phi\right]_{i, j, k, m}^{n+1}+\mathcal{R}^{n+1} \rho_{i n s} S_{i}^{j}-\mathcal{M}^{n+1} f_{i, j, k}^{n+1} \geq 0 .}{(\mathrm{F} .26)}
\end{array}
$$

Since $\epsilon>0$, equation (F.25) is equivalent to:

$$
\epsilon\left(\frac{\phi_{i, j, k, m}^{n+1}-\phi_{i, j, k, m}^{n}}{\Delta \tau}-\left[\mathcal{L}^{h} \phi\right]_{i, j, k, m}^{n+1}+\mathcal{R}^{n+1} \rho_{i n s} S_{i}^{j}-\mathcal{M}^{n+1} f_{i, j, k}^{n+1}\right)+\phi_{i, j, k, m}^{n+1}-\left[\mathcal{A}^{h} \phi\right]_{i, j, k, m}^{n+1} \geq 0 .
$$

Similarly, equations (F.26) and (F.27) can be combined to obtain:

$$
\begin{gathered}
\min \left(\epsilon\left(\frac{\phi_{i, j, k, m}^{n+1}-\phi_{i, j, k, m}^{n}}{\Delta \tau}-\left[\mathcal{L}^{h} \phi\right]_{i, j, k, m}^{n+1}+\mathcal{R}^{n+1} \rho_{i n s} S_{i}^{j}-\mathcal{M}^{n+1} f_{i, j, k}^{n+1}\right)+\phi_{i, j, k, m}^{n+1}-\left[\mathcal{A}^{h} \phi\right]_{i, j, k, m}^{n+1},\right. \\
\left.\frac{\phi_{i, j, k, m}^{n+1}-\phi_{i, j, k, m}^{n}}{\Delta \tau}-\left[\mathcal{L}^{h} \phi\right]_{i, j, k, m}^{n+1}+\mathcal{R}^{n+1} \rho_{i n s} S_{i}^{j}-\mathcal{M}^{n+1} f_{i, j, k}^{n+1}\right)=0
\end{gathered}
$$

which corresponds to the definition of $\hat{\mathcal{G}}\left(h, x, V_{i, j, k, m}^{n+1}, V_{i, j, k, m}^{n},\left\{V_{a, p, u, l}^{n+1}\right\}\right)$ in equation (6.13) for interior nodes. Applying the same technique for the boundary nodes, we can show the equivalence between the original scheme $\mathcal{G}\left(h, x, V_{i_{\max }, j, k, m}^{n+1}, V_{i_{\max , j, k, m}}^{n},\left\{V_{a, p, u, l}^{n+1}\right\}\right)$ in equation (6.7) and $\hat{\mathcal{G}}\left(h, x, V_{i_{\max }, j, k, m}^{n+1}, V_{i_{\max }, j, k, m}^{n},\left\{V_{a, p, u, l}^{n+1}\right\}\right)$ in equation (6.14). This demonstration is omitted for brevity. 


$$
\begin{aligned}
& \left|\hat{\mathcal{G}}\left(h, x, \phi_{i, j, k, m}^{n+1}+\xi, \phi_{i, j, k, m}^{n}+\xi,\left\{\phi_{a, p, u, l}^{n+1}+\xi\right\}\right)-F_{i n}(\phi)_{i, j, k, m}^{n+1}\right| \\
& \leq \max \left(\mid\left(\phi_{i, j, k, m}^{n+1}+\xi-\mathcal{A}^{h}\left(\phi_{i, j, k, m}^{n+1}+\xi\right)\right)-[\phi-\mathcal{A} \phi]_{i, j, k, m}^{n+1}\right. \\
& \quad+\epsilon\left(\frac{\phi_{i, j, k, m}^{n+1}-\phi_{i, j, k, m}^{n}}{\Delta \tau}-\left[\mathcal{L}^{h}(\phi+\xi)\right]_{i, j, k, m}^{n+1}+\mathcal{R}^{n+1} \rho_{i n s} S_{i}^{j}-\mathcal{M}^{n+1} f_{i, j, k}^{n+1}\right) \mid \\
& \quad \mid\left(\frac{\phi_{i, j, k, m}^{n+1}-\phi_{i, j, k, m}^{n}}{\Delta \tau}-\left[\mathcal{L}^{h}(\phi+\xi)\right]_{i, j, k, m}^{n+1}+\mathcal{R}^{n+1} \rho_{i n s} S_{i}^{j}-\mathcal{M}^{n+1} f_{i, j, k}^{n+1}\right) \\
& \left.\quad-\left[\phi_{\tau}-\mathcal{L} \phi+\mathcal{R}(\tau) \rho_{i n s} S-\mathcal{M}(\tau) f\right]_{i, j, k, m}^{n+1} \mid\right) \\
& =\max \left(\mid O\left(\Delta S_{\max }^{j} \Delta B_{\max }^{u}\right)+O\left(h^{2}\right)+\epsilon\left(\frac{\phi_{i, j, k, m}^{n+1}-\phi_{i, j, k, m}^{n}}{\Delta \tau}-\left[\mathcal{L}^{h} \phi\right]_{i, j, k, m}^{n+1}+\mathcal{R}^{n+1} \rho_{i n s} S_{i}^{j}\right.\right. \\
& \left.\left.-\mathcal{M}^{n+1} f_{i, j, k}^{n+1}-\xi r\right)|,| O(\Delta \tau)+O\left(\Delta S_{\max }^{j}\right)+r \xi \mid\right)
\end{aligned}
$$


Similarly, for the boundary nodes when $S_{i}^{j}=S_{\max }$, we have:

$$
\begin{aligned}
& \left|\hat{\mathcal{G}}\left(h, x, \phi_{i_{\max }, j, k, m}^{n+1}+\xi, \phi_{i_{\max }, j, k, m}^{n}+\xi,\left\{\phi_{a, p, u, l}^{n+1}+\xi\right\}\right)-F_{\text {bound }}(\phi)_{i_{\max }, j, k, m}^{n+1}\right| \\
& \leq \max \left(\mid\left(\phi_{i, j, k, m}^{n+1}+\xi-\mathcal{A}^{h}\left(\phi_{i, j, k, m}^{n+1}+\xi\right)\right)-[\phi-\mathcal{A} \phi]_{i, j, k, m}^{n+1}+\epsilon\left(\frac{\phi_{i_{\max }, j, k, m}^{n+1}-\phi_{i_{\max }, j, k, m}^{n}}{\Delta \tau}\right.\right. \\
& \left.+\rho_{\text {total }}\left(\phi_{i_{\max }, j, k, m}^{n+1}+\xi\right)-\sum_{\substack{l=1 \\
l \neq m}}^{M} \lambda^{m \rightarrow l} J_{i_{\max }}^{m \rightarrow l}\left(\phi_{i_{\max }, j, k, l}^{n+1}-\phi_{i_{\max }, j, k, m}^{n+1}\right)+\mathcal{R}^{n+1} \rho_{i n s} S_{i_{\max }}^{j}\right) \mid, \\
& \mid\left(\frac{\phi_{i_{\max }, j, k, m}^{n+1}-\phi_{i_{\max , j, k, m}^{n}}^{n}}{\Delta \tau}+\rho_{\mathrm{total}}\left(\phi_{i_{\max }^{n}, j, k, m}^{n+1}+\xi\right)-\sum_{\substack{l=1 \\
l \neq m}}^{M} \lambda^{m \rightarrow l} J_{i_{\max }}^{m \rightarrow l}\left(\phi_{i_{\max }, j, k, l}^{n+1}-\phi_{i_{\max }, j, k, m}^{n+1}\right)\right. \\
& \left.\left.+\mathcal{R}^{n+1} \rho_{\text {ins }} S_{i_{\max }}^{j}\right)-\left[\phi_{\tau}+\rho_{\text {total }} \phi-\sum_{\substack{l=1 \\
l \neq m}}^{M} \lambda^{m \rightarrow l} J^{m \rightarrow l}(S)\left(\phi\left(S, B, D, e_{l}, \tau\right)-\phi\right)+\mathcal{R}(\tau) \rho_{\text {ins }} S\right]_{i_{\max }, j, k, m}^{n+1} \mid\right) \\
& =\max \left(\mid O\left(\Delta S_{\max }^{j} \Delta B_{\max }^{u}\right)+O\left(h^{2}\right)+\epsilon\left(\frac{\phi_{i_{\max }^{n+1}, j, k, m}^{n+1}-\phi_{i_{\max }, j, k, m}^{n}}{\Delta \tau}\right.\right. \\
& \left.+\rho_{\text {total }}\left(\phi_{i_{\max }, j, k, m}^{n+1}+\xi\right)-\sum_{\substack{l=1 \\
l \neq m}}^{M} \lambda^{m \rightarrow l} J_{i_{\max }}^{m \rightarrow l}\left(\phi_{i_{\max }, j, k, l}^{n+1}-\phi_{i_{\max }, j, k, m}^{n+1}\right)+\mathcal{R}^{n+1} \rho_{i n s} S_{i_{\max }}^{j}\right) \mid, \\
& \left.\left|O(\Delta \tau)+O\left(\Delta S_{\max }^{j}\right)+\rho_{\text {total }} \xi\right|\right) .
\end{aligned}
$$

Using Assumption 6.6, we obtain:

$$
\hat{\mathcal{G}}\left(h, x, \phi_{i, j, k, m}^{n+1}+\xi, \phi_{i, j, k, m}^{n}+\xi,\left\{\phi_{a, p, u, l}^{n+1}+\xi\right\}\right)=F(\phi)_{i, j, k, m}^{n+1}+O(h)+\xi b(x),
$$

for both boundary and interior nodes, where $b(x)$ is a bounded function with $|b(x)| \leq \max \left(r, \rho_{\text {total }}\right)$.

We now present the proof of Theorem 6.13.

Proof. (of Theorem 6.13)

We begin by proving that equation (6.16) holds. From the definition of limsup, there exists sequences $h_{d}, i_{d}, j_{d}, k_{d}, n_{d}, \xi_{d}$ such that

$$
h_{d} \rightarrow 0, \xi_{d} \rightarrow 0, x_{d}=\left(S_{i_{d}}^{j_{d}}, B_{j_{d}}^{k_{d}}, D_{k_{d}}, e_{m}, \tau^{n_{d}+1}\right) \rightarrow \hat{x}=\left(\hat{S}, \hat{B}, \hat{D}, e_{m}, \hat{\tau}\right) \text { as } d \rightarrow \infty
$$

and

$$
\begin{array}{r}
\lim \sup _{d \rightarrow \infty} \hat{\mathcal{G}}\left(h_{d}, x_{d}, \phi_{i_{d}, j_{d}, k_{d}, m}^{n_{d}+1}+\xi_{d}, \phi_{i_{d}, j_{d}, k_{d}, m}^{n_{d}}+\xi_{d},\left\{\phi_{a_{d}, p_{d}, u_{d}, l}^{n_{d}+1}+\xi_{d}\right\}\right) \\
=\lim \sup _{\substack{\xi, h \rightarrow 0 \\
x \rightarrow \hat{x}}} \hat{\mathcal{G}}\left(h, x, \phi_{i, j, k, m}^{n+1}+\xi, \phi_{i, j, k, m}^{n}+\xi,\left\{\phi_{a, p, u, l}^{n+1}+\xi\right\}\right) .
\end{array}
$$


From our result in equation (F.5), we have:

$$
\hat{\mathcal{G}}\left(h_{d}, x_{d}, \phi_{i_{d}, j_{d}, k_{d}, m}^{n_{2}+1}+\xi_{d}, \phi_{i_{d}, j_{d}, k_{d}, m}^{n_{d}}+\xi_{d},\left\{\phi_{a_{d}, p_{d}, u_{d}, l}^{n_{d}+1}+\xi_{d}\right\}\right)=F\left(\phi\left(x_{d}\right)\right)+O\left(h_{d}\right)+\xi_{d} b\left(x_{d}\right),
$$

where $F(\phi(x))$ is defined in equation (6.10) for interior and boundary nodes.

Now consider a sequence of nodes $x_{d}$ as defined in equation (F.32) which may contain both interior $\left(S_{i_{d}}^{j_{d}}<S_{\max }\right)$ and boundary nodes $\left(S_{i_{d}}^{j_{d}}=S_{\max }\right)$. Combining equation (F.34) with equation (F.33), we get:

$$
\begin{aligned}
\lim \sup _{\substack{\xi, h \rightarrow 0 \\
x \rightarrow \hat{x}}} \hat{\mathcal{G}}\left(h, x, \phi_{i, j, k, m}^{n+1}+\xi, \phi_{i, j, k, m}^{n}+\xi,\left\{\phi_{a, p, u, l}^{n+1}+\xi\right\}\right) \\
\quad \leq \lim \sup _{d \rightarrow \infty} F\left(\phi\left(x_{d}\right)\right)+\lim \sup _{d \rightarrow \infty}\left[O\left(h_{d}\right)+\xi_{d} b\left(x_{d}\right)\right] \leq F^{*}(\phi(\hat{x}))
\end{aligned}
$$

where the last inequality holds because of:

$$
\lim \sup _{d \rightarrow \infty}\left[O\left(h_{d}\right)+\xi_{d} b\left(x_{d}\right)\right]=0 .
$$

Verifying equation (6.17) can be done in a similar fashion.

Having shown that equations (6.16) and (6.17) hold, we conclude that the discrete equations in (5.3) are consistent according to Definition 6.11.

\section{G Comparison with Previous GMDB Numerical Results}

In [33], the authors present an analytical model to price GMDB contracts with different death benefit guarantees including return-of-premium, rising floor and ratchets. More specifically, the authors determine the fair insurance charge that equates the present value of the risk charges with the value of the death benefit guarantee. While mostly focusing on guarantees with a rising floor, basic numerical results for contracts with a continuous lookback or ratchet feature are included in [33]. To validate the GMDB pricing model presented in Section 2, we attempt to reproduce the numerical results presented in [33] when valuing a GMDB clause with ratchets.

For consistency with the problem considered in [33], we modify the GMDB pricing problem presented in Section B to satisfy the following:

- Since the authors of [33] focus on determining the value for $\rho_{\text {ins }}$, the contract considered does not include any management fees. Consequently, we set $\rho_{\text {man }}=0$.

- The contract considered in [33] does not include the partial withdrawal or lapsing feature. Thus, we will solve the following pricing equation:

$$
V_{\tau}=\frac{1}{2} \sigma^{2} S^{2} V_{S S}+\left(r-\rho_{i n s}\right) S V_{S}-r V-\mathcal{R}(\tau) \rho_{\text {ins }} S+\mathcal{M}(\tau) \max (B-S, 0),
$$

without imposing an impulse control.

- To reproduce the continuous ratchet assumption, we apply the update feature presented in equation (4.8) discretely at each timestep during the solution process. As $\Delta \tau \rightarrow 0$, the value of the GMDB guarantee will converge to the contract value with continuous ratchets. 


\begin{tabular}{|c|c|c|c|c|c|}
\hline \multicolumn{6}{|c|}{ GMDB with Continuous Ratchet } \\
\hline \multirow[b]{2}{*}{ Refinement } & \multirow[b]{2}{*}{ Timesteps } & \multicolumn{2}{|c|}{ Nodes } & \multicolumn{2}{|c|}{ Insurance Charge - $\rho_{\text {ins }}$} \\
\hline & & $S$ & $B$ & Male & Female \\
\hline 0 & 2500 & 80 & 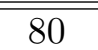 & 0.003960 & 0.002334 \\
\hline 1 & 5000 & 159 & 159 & 0.004042 & 0.002383 \\
\hline 2 & 10000 & 317 & 314 & 0.004086 & 0.002409 \\
\hline 3 & 20000 & 633 & 633 & 0.004114 & 0.002426 \\
\hline 4 & 40000 & 1265 & 1265 & 0.004133 & 0.002437 \\
\hline \multicolumn{4}{|c|}{ Value from $[33]$} & 0.00418 & 0.00246 \\
\hline
\end{tabular}

Table G.1: Fair insurance charge $\rho_{\text {ins }}$ for a GMDB contract with discrete ratchet events when the owner is assumed to be 50 years old when the contract is purchased. The contract assumptions are chosen to approximate those in [33]. Crank-Nicolson timestepping with constant timesteps was used. We assume $\sigma=0.20, r=0.06, \rho_{\text {man }}=0$ and set the initial timestep is set to $\Delta \tau=0.01$ years on the coarsest grid.

In [33], the authors assume that the contract terminates when the owner is 75 years old and consider a range of values for the age of the contract owner at the time of purchase (namely 30,40,50,60 and 65 years old). We will focus our analysis on the case most similar to the rest of the results in this paper and assume that the contract owner is 50 years old at the time of purchase; this implies that $T=25$ years in our pricing model.

To be consistent with [33], we set $\sigma=0.20$ and $r=0.06$. In addition, the mortality data is generated with a Gompertz mortality distribution using the parameters presented in [33] corresponding to the age of the contract owner when the contract is purchased. The parameters in [33] are obtained by fitting a Gompertz mortality distribution to the 1994 Group Annuity Mortality Table (Basic) over the contract lifetime. In our case, we approximate the continuous mortality function with a discrete mortality distribution generated with $\Delta \tau=6.25 \times 10^{-4}$ years. Such a small $\Delta \tau$ is chosen to avoid interpolation issues for higher refinement levels.

Recall that we are looking to determine the fair insurance charge $\rho_{\text {ins }}$ that satisfies:

$$
V\left(\rho_{\text {ins }} ; S=\$ 100, B=\$ 100, D=\$ 100, \tau=T\right)=0 .
$$

Newton iteration is used during the solution process and the tolerance is set to $1 \times 10^{-6}$. The resulting insurance charges are presented in Table G.1.

We see that the results obtained in Table G.1 are consistent with those presented in [33] but exhibit slow convergence. Keep in mind that the authors of [33] generate their results with analytical formulas while we approximate the contract considered by using discrete ratchet events. In Table G.1, we are essentially valuing a discrete lookback option which is a difficult problem. As the ratchet interval is reduced, the value of a discrete lookback is known to converge very slowly to the corresponding continuous lookback value $[3,13,29]$.

Nonetheless, the numerical results in Table G.1 are certainly sufficient for practical purposes. Similar levels of accuracy were observed when comparing our numerical results to the analytical values in [33] for the remaining cases (i.e. when the owner is assumed to be 30,40,60 and 65 years old). 


\section{References}

[1] A. L. Amadori. Quasi-variational inequalities with Dirichlet boundary conditions related to exit time problems for impulse control. SIAM Journal on Control and Optimization, 43(2):570$589,2004$.

[2] E. Ayache, P. Henrotte, S. Nassar, and X. Wang. Can anyone solve the smile problem? Wilmott Magazine, pages 78-96, January 2004.

[3] S. Babbs. Binomial valuation of lookback options. Journal of Economic Dynamics and Control, 24:1499-1525, 2000.

[4] G. Barles. Numerical Methods in Finance, chapter Convergence of Numerical Schemes for Degenerate Parabolic Equations Arising in Finance Theory, pages 1-21. Cambridge University Press, 1997.

[5] G. Barles and E. Jakobsen. Error bounds for monotone approximation schemes for parabolic Hamilton-Jacobi-Bellman equations. Mathematics of Computation, 76:1861-1893, 2007.

[6] G. Barles and P. E. Souganidis. Convergence of approximation schemes for fully nonlinear equations. Asymptotic Analysis, 4:271-283, 1991.

[7] D. Bauer, A. Kling, and J. Russ. A universal pricing framework for guaranteed minimum benefits in variable annuities. Working paper, Ulm University, 2006.

[8] N. P. B. Bollen. Valuing options in regime-switching models. Journal of Derivatives, 6(1):3849, 1998.

[9] P. Boyle and T. Draviam. Pricing exotic options under regime switching. Insurance: Mathematics and Economics, 40(2):267-282, 2007.

[10] G. Briere-Giroux, D. Czernicki, D. Maloof, and H. Mueller. Variable annuity products face tough decisions in the midst of the financial crisis. working paper, Towers Perrin, 2008.

[11] J. Buffington and R. J. Elliott. American options with regime-switching. International Journal of Theoretical and Applied Finance, 5(5):497-514, 2002.

[12] Z. Chen and P. A. Forsyth. A numerical scheme for the impulse control formulation for pricing variable annuities with a guaranteed minimum withdrawal benefit (GMWB). Working paper, University of Waterloo, Cheriton School of Computer Science, 2007.

[13] T. Cheuk and T. Vorst. Currency lookback options and observation frequency: A binomial approach. Journal of International Money and Finance, 16(2):173-187, 1997.

[14] S. Choi. Regime-switching univariate diffusion models of the short-term interest rate. Working paper, School of Economics, University of Adelaide, Australia, 2004.

[15] T. F. Coleman, Y. Li, and M. Patron. Hedging guarantees in variable annuities (under both market and interest rate risks). Insurance: Mathematics and Economics, 38:215-228, 2006. 
[16] Collection of Life Tables - Recueil des tables de mortalité canadiennes - 1801-1996. CIED (Centre interuniversitaire d'études démographiques), Department of demography, University of Montreal, June 2003.

[17] R. Cont and P. Tankov. Financial Modelling with Jump Processes. Chapman \& Hall / CRC, 2004 .

[18] E. Cramer, P. Matson, and L Rubin. Common practices relating to FASB statement 133, Accounting for Derivative Instruments and Hedging Activities as it Relates to Variable Annuities with Guaranteed Benefits. Practice Note, American Academy of Actuaries, 2007.

[19] M. G. Crandall, H. Ishii, and P.-L. Lions. User's guide to viscosity solutions of second order differential equations. Bulletin of the American Mathematical Society, 27:1-67, July 1992.

[20] M. Dai, Y. K. Kwok, and J. Zong. Guaranteed minimum withdrawal benefit in variable annuities. To appear in Mathematical Finance, 2007.

[21] J.-C. Duan, I. Popova, and P. Ritchken. Option pricing under regime switching. Quantitative Finance, 2:116-132, 2002.

[22] P. A. Forsyth and G. Labahn. Numerical methods for controlled Hamilton-Jacobi-Bellman PDEs in finance. Journal of Computational Finance, 11 (Winter)(2):1-44, 2008.

[23] P. A. Forsyth and K. R. Vetzal. Quadratic convergence for valuing American options using a penalty method. SIAM Journal on Scientific Computing, 23:2095-2122, 2002.

[24] C. Frantz, X. Chenut, and J. Walhin. Pricing and capital allocation for unit-linked life insurance contracts with minimum death guarantee. Proceedings of the AFIR Colloquium, 2003.

[25] Toronto Globe and Mail. Manulife, in red, raises new equity. December 2, 2008.

[26] S. F. Gray. Modeling the conditional distribution of interest rates as a regime-switching process. Journal of Financial Economics, 42:27-62, 1996.

[27] J. D. Hamilton. A new approach to the economic analysis of non-stationary time series. Econometrica, 57:357-384, 1989.

[28] M. R. Hardy. A regime-switching model of long-term stock returns. North American Actuarial Journal, 5(2):41-53, 2001.

[29] J. C. Hull. Options, Futures, and Other Derivatives. Prentice Hall, Upper Saddle River, NJ, 5th edition, 2003.

[30] K. Ishii. Viscosity solutions for nonlinear second order elliptic PDEs associated with impulse control problems II. Funkcialaj Ekvacioj, 38:297-328, 1995.

[31] R. Korn. Some applications of impulse control in mathematical finance. Mathematics of Operations Research, 50:493-518, 1999.

[32] W. Liu, Y. Yang, and G. Lu. Viscosity solutions of fully nonlinear parabolic systems. Journal of Mathematical Analysis and Applications, 281:362-381, 2002. 
[33] M. Milevsky and S. Posner. The Titanic Option: Valuation of the guaranteed minimum death benefit in variable annuities and mutual funds. Journal of Risk and Insurance, 68(1):91-126, 2001 .

[34] M. A. Milevsky and T. S. Salisbury. Financial valuation of guaranteed minimum withdrawal benefits. Insurance: Mathematics and Economics, 38(1):21-38, 2006.

[35] B. Mudavanhu and J. Zhuo. Valuing guaranteed minimum death benefits in variable annuities and the option to lapse. Submitted to the North American Actuarial Journal, March 2002.

[36] M. Pemy and Q. Zhang. Optimal stock liquidation in a regime switching model with finite time horizon. Journal of Mathematical Analysis and Applications, 321:537-552, 2006.

[37] P. M. Philipps. Senior Vice President and Director, CIGNA Reinsurance. Private communication, 2004.

[38] D. M. Pooley, P. A. Forsyth, and K. R. Vetzal. Numerical convergence properties of option pricing PDEs with uncertain volatility. IMA Journal on Numerical Analysis, 23:241-267, 2003.

[39] R. Rannacher. Finite element solution of diffusion problems with irregular data. Numerische Mathematik, 43:309-327, 1984.

[40] V. L. Vath, M. Mnif, and H. Pham. A model of optimal portfolio selection under liquidity risk and price impact. Finance and Stochastics, 11:51-90, 2007.

[41] P. Wilmott. Derivatives: The Theory and Practice of Financial Engineering. Wiley, West Sussex, England, 1998.

[42] H. Windcliff. Computational methods for valuing path-dependent derivatives. PhD thesis, University of Waterloo, 2003.

[43] H. Windcliff, P. A. Forsyth, and K. R. Vetzal. Valuation of segregated funds: Shout options with maturity extensions. Insurance: Mathematics and Economics, 29(1):1-21, 2001.

[44] H. Windcliff, P. A. Forsyth, and K. R. Vetzal. Analysis of the stability of the linear boundary condition for the Black-Scholes equation. Journal of Computational Finance, 8(1):65-92, 2004.

[45] S. Wu and Y. Zeng. A general equilibrium model of the term structure of interest rates under regime-switching risk. International Journal of Theoretical and Applied Finance, 8(7):839-869, 2005 .

[46] D. D. Yao, Q. Zhang, and X. Y. Zhou. A regime-switching model for European Option Pricing, chapter Stochastic Processes, Optimization, and Control Theory: Applications in Financial Engineering, Queuing Networks, and Manufacturing Systems, pages 281-300. Springer USA, 2006. 